\title{
Comparative review of endoscopic devices articulations technologies developed for minimally invasive medical procedures
}

\author{
Julien Catherine $^{\mathrm{a}, *}$, Christine Rotinat-Libersa ${ }^{\mathrm{a}}$ and Alain Micaelli ${ }^{\mathrm{b}}$ \\ ${ }^{a}$ CEA, LIST, Interactive Robotics Laboratory, Fontenay aux Roses, France \\ ${ }^{\mathrm{b}}$ CEA, LIST, Interactive Simulation Laboratory, Fontenay aux Roses, France
}

\begin{abstract}
This study introduces a comparative performance analysis of the technological solutions that have been used to build distal active articulations for minimally invasive medical procedures. The aim is to provide a practical and concise database and classification tool for anyone that wants to learn more about the technologies involved in minimally invasive medical devices, or for any designer interested in further improving these devices. A review of the different articulations developed in this field is therefore performed and organized by both actuation technology and structural architecture. Details are presented concerning the mechanical structures as well as the actuation and the mechanical transmission technologies available. The solutions are evaluated keeping as a reference some chosen required performances and characteristics for minimally invasive surgical procedures. Finally, a quantified comparison chart of these devices is given regarding selected criteria of interest for minimally invasive surgical application.
\end{abstract}

Keywords: Minimally invasive procedures, endoscopic devices, articulation, actuation technology

\section{Introduction}

The absolute goal of medicine is to maintain and restore health of people without causing any side effects or injuries. Minimally invasive medical procedures (MIP) are going towards this goal by reducing the dimensions of the incisions necessary to access targeted tissues for treatment or examination. Indeed, these techniques allow the realization of an increasing number of surgical procedures and medical examinations with an access to the targeted tissues through several incisions of a few millimetres, through one sin-

*Corresponding author: Julien Catherine, CEA, LIST, Interactive Robotics Laboratory, 18, route du Panorama, BP6, Fontenay Aux Roses, F-92265, France. Tel.: +33 1465497 29; Fax: +33 1465489 80. E-mail: julien.catherine@cea.fr, Julien.catherine@ gmail.com. gle incision with Single Input Laparoscopic Surgery (SILS), or even through natural orifices and internal incisions with the emerging technique of Natural Orifice Transluminal Endoscopic Surgery (NOTES). This invasiveness reduction resulting effectively in strong medical, economical and aesthetical benefits, MIP mean to be performed in an increasing number of cases in domains as varied as orthopaedics, gynecology, urology, cancerology, and thoracic, cardiac or neurosurgery.

This became possible thanks to specific technological advances in domains as varied as medical imaging, computer assistance and design of medical devices that allowed overcoming some drawbacks induced by the small dimensional access. Actually, the main challenge for further development of MIP is, along with reducing invasiveness, to allow the physician to be as efficient 
as in open procedures by ensuring the required control, dexterity, force generation, freedom of movements through the whole procedures.

Focusing on the challenging minimally invasive surgery (MIS) and in comparison to early manually driven MIS instruments, computer assisted systems managed to give back to the surgeon comfort and part of the freedom of movements of open surgery. These robotic systems also suppress the inversion of movements due to the fulcrum at the insertion point and allow the surgeon to control the endoscope by himself. Some further improvements such as tremor filtering and homothetic reduction of the surgeon's movements have been made possible as well.

Nevertheless, drawbacks can still be identified in the nowadays clinically used MIS computer assisted systems [13] whose dexterity and freedom of movements are still limited and incompatible with some surgical procedures. Regarding the daVinci ${ }^{\circledR}$ surgical robot (developed by Intuitive Surgical ${ }^{\circledR}$ ), which is the most common surgical robotic system used in clinics, its major drawback is that the access to some sites inside the body is still impossible or very difficult due to the robotic arms rigid shafts even by optimizing the positions of the trocars. Moreover, it is still hazardous to change surgical sites during the operation without modifying the location of the trocars and performing new incisions. It is therefore essentially used for urology or digestive system surgery procedures, where its benefits have been proven [32].

In order to execute more complex MIS at sites that are more difficult to be reached inside the body, key components of medical instruments are the articulations producing the intracorporal mobilities to convey the tools through the body towards the surgical site and manipulate them. Such articulations were missing on the first laparoscopic instruments but have been progressively added and have evolved to enable movements with several degrees of freedom (DOF) and of larger amplitudes. Despite these evolutions, there is still a need for further miniaturized articulations enabling larger displacements, with the proper force performance and dynamics to perform MIS as efficiently as open surgery.

The present study gives an overview of the different intracorporal articulations developed in the field of MIP, in order to manipulate surgical tools or to simply investigate tissues. Commercialized and already clinically used articulated devices are studied separately from those that are still not validated for use on human patients. A distinction is then done between systems with a deported actuation needing a mechanical transmission and the ones with local actuation. Depending on the category, a more refined classification is introduced depending on the structural architecture and the actuation technology for locally actuated systems. The study of their respective performances and characteristics, keeping as a reference the MIS required once, will enable to evaluate and compare them, as well as the technologies associated, in order to help choosing the proper technology for the conception of efficient robotic systems for this particular application field. The comparison will be based on different characteristics such as the size of the articulation (i.e. external diameter and length), which is of main interest in order to further reduce the size of the incisions for minimally invasive procedures. The number of degrees of freedom, the amplitude of movement along the DOFs and the radius of curvature are criteria for characterizing the capacity of the articulation to drive the device inside the body while avoiding organs, and to follow an unexpected spatial trajectory without changing the insertion point. They describe as well the possibility to control the medical instruments to perform complicated gestures in a very limited workspace. The torque generated by the articulation is also given when available, and is important to perform surgical tasks such as suturing. Other criteria, such as resolution, reliability, accuracy (and others) of the systems and characteristics of their power supply (e.g. amplitude of fluid pressure, electrical current or voltage for instance) will not be given here due to a lack of data in the explored literature. Furthermore, the fact that the surgeon always keeps control on the device, and therefore close the control loop, reduce the importance of criteria such as the accuracy of the system, that can be corrected in real time by the surgeon. Available numerical values of performances and characteristics are not always given in the text but they are grouped in Table 1 .

\section{Review of the articulation technologies used in commercially available minimally invasive devices}

As far as the investigating authors know, every device certified for clinical use that offers many DOFs, more than the simple control of the distal tool, present intracorporal articulations with externalized actuation and mechanical transmission using mechanical cables, 
Table 1

Values of the chosen criteria for each device referenced. Articulations are named by their related reference. Information on their characteristics are notified in the first column after the reference in brackets as following: (a) refers to modular systems; (b) refers to an estimated length after removing the passive structural parts length; (c) refers to an estimated length; (d) refers to an estimated radius of curvature; (e) refers to an

\begin{tabular}{|c|c|c|c|c|c|c|c|c|}
\hline Articulation & $\begin{array}{l}\text { Actuation/ } \\
\text { transmission } \\
\text { technology }\end{array}$ & $\begin{array}{l}\text { Modular structure } \\
\text { (Number of } \\
\text { modules - } \\
\text { Number of DOFs } \\
\text { per modules) }\end{array}$ & $\begin{array}{l}\text { Diameter } \\
(\mathrm{mm})\end{array}$ & $\begin{array}{l}\text { Length } \\
(\mathrm{mm})\end{array}$ & $\begin{array}{l}\text { Bending } \\
\text { angle } \\
\text { (deg) }\end{array}$ & $\begin{array}{c}\text { Radius } \\
\text { of (mm) } \\
\text { curvature }\end{array}$ & $\begin{array}{l}\text { Number } \\
\text { of DOFs }\end{array}$ & $\begin{array}{l}\text { Torque } \\
(\mathrm{N} \mathrm{mm})\end{array}$ \\
\hline $\begin{array}{l}\text { Reference } \\
\text { performances }\end{array}$ & & yes $(1-4)$ & 3 & 5 & 90 & 1.5 & 4 & 150 \\
\hline$[53]-(\mathrm{d}, \mathrm{c})$ & Connecting rods & no & 6.4 & 15 & 180 & 4.8 & 1 & - \\
\hline$[43]-(d, c)$ & Connecting rods & no & 20 & 30 & 45 & 30 & 3 & - \\
\hline [59] $-(d, c)$ & Connecting rods & no & 9 & 50 & 85 & 16.8 & 2 & 207 \\
\hline [60] - (c) & Connecting rods & no & 3.5 & 15 & 90 & 3.8 & 2 & 27.9 \\
\hline [58] - (e) & $\begin{array}{c}\text { Flexible } \\
\text { structures }\end{array}$ & no & 42 & 28 & 73.5 & 21.8 & 2 & 15 \\
\hline$[35]-(d, c)$ & Mechanical & no & 8.8 & 45 & 210 & 12.3 & 2 & - \\
\hline [29] $-(d, c)$ & Mechanical cables & no & 5 & 13 & 90 & 8.3 & 3 & - \\
\hline [57] $-(\mathrm{d}, \mathrm{c})$ & Mechanical & no & 4.7 & 48 & 270 & 10.1 & 8 & - \\
\hline [8] - (d) & Mechanical cables & no & 5 & 30 & 90 & 9.5 & 2 & 250 \\
\hline [37] - (a) & Mechanical cables & yes $(-2)$ & 12 & 12 & 10 & 35 & 2 & - \\
\hline$[31]-(\mathrm{d}, \mathrm{c})$ & Mechanical cables & no & 8 & 25 & 90 & 12.5 & 2 & - \\
\hline$[56]-(d, b)$ & Mechanical cables & no & 10 & 55 & 90 & 12.5 & 4 & 530 \\
\hline$[42]-(\mathrm{d}, \mathrm{c})$ & Mechanical cables & no & 8 & 45 & 90 & 8 & 3 & - \\
\hline$[48]-(\mathrm{d}, \mathrm{c}, \mathrm{e})$ & Mechanical cables & no & 10 & 20 & 40 & 27 & 2 & 600 \\
\hline [7] - (d,e) & Mechanical cables & no & 10 & 20 & 45 & 25 & 2 & 325 \\
\hline [26] & Mechanical cables & no & 10 & - & - & - & 6 & 14 \\
\hline [4] - (c) & Mechanical cables & no & 5 & 17.5 & 110 & 8 & 2 & - \\
\hline [41] - (d) & Mechanical cables & no & 4.9 & 22.5 & 90 & 14.3 & 2 & - \\
\hline$[54]-(d, e)$ & Mechanical cables & no & 6 & 8 & 85 & 5.4 & 2 & 150 \\
\hline [18] - (d) & Mechanical cables & no & 2.4 & 19.9 & 180 & 6.3 & 2 & - \\
\hline$[50]-(d, e)$ & Fluidic & no & 4 & 20 & 95 & 12 & 3 & 1.5 \\
\hline [6] & Fluidic+SMA & no & 4 & 40 & 90 & 25.5 & 2 & - \\
\hline [45] - (d) & Fluidic & no & 5 & 4 & 5 & 114 & 2 & - \\
\hline$[27]-(d)$ & Fluidic & no & 24 & 13 & 45 & 16.5 & 3 & - \\
\hline [2] - (d) & Fluidic & no & 4.9 & 20 & 35 & 32.7 & 3 & - \\
\hline$[40]-(d)$ & Fluidic & no & 12 & 30 & 35 & 10 & 3 & 20 \\
\hline [22] - (a) & Fluidic & yes $(1-1)$ & 1.5 & 40 & - & - & 1 & - \\
\hline [16] & Fluidic & no & 0.9 & 5.5 & 160 & 2 & 1 & - \\
\hline$[25]-(d, a)$ & SMA & yes $(5-2)$ & 13 & 215 & 300 & 41.8 & 10 & - \\
\hline$[25]-(d, a)$ & SMA & yes $(1-2)$ & 13 & 43 & 60 & 41.8 & 2 & - \\
\hline [51] & SMA & no & 1.5 & 20 & 45 & 25.5 & 2 & - \\
\hline$[5]-(d)$ & SMA & no & 3 & 40 & 90 & 25.5 & 2 & - \\
\hline$[38]-(\mathrm{d}, \mathrm{c}, \mathrm{a})$ & SMA & yes $(3-2)$ & 2 & 36 & 147 & 14 & 6 & - \\
\hline
\end{tabular}

mechanical rods or a flexible structure. The different types of articulations that can be encountered are presented here.

\subsection{Cable-driven distributed articulations}

This configuration is the most commonly integrated in devices used clinically for procedures requiring sev- eral local DOFs or bending with large amplitude. It is composed of a bendable structure along 1 or 2 DOFs (numerous joints of limited bending capabilities or compliant structure) driven by cables acting as tendons in a finger. A large number of such devices are manually driven, with a handle very close to classical laparoscopic instruments but with more controls for the added DOFs. Several companies, such as Olympus 


\begin{tabular}{|c|c|c|c|c|c|c|c|c|}
\hline & & & $\begin{array}{l}\text { Table } 1 \\
\text { ontinued) }\end{array}$ & & & & & \\
\hline Articulation & $\begin{array}{l}\text { Actuation/ } \\
\text { transmission } \\
\text { technology }\end{array}$ & $\begin{array}{l}\text { Modular structure } \\
\text { (Number of } \\
\text { modules - } \\
\text { Number of DOFs } \\
\text { per modules) }\end{array}$ & $\begin{array}{l}\text { Diameter } \\
(\mathrm{mm})\end{array}$ & $\begin{array}{l}\text { Length } \\
(\mathrm{mm})\end{array}$ & $\begin{array}{l}\text { Bending } \\
\text { angle } \\
\text { (deg) }\end{array}$ & $\begin{array}{c}\text { Radius } \\
\text { of (mm) } \\
\text { curvature }\end{array}$ & $\begin{array}{l}\text { Number } \\
\text { of DOFs }\end{array}$ & $\begin{array}{l}\text { Torque } \\
(\mathrm{N} \mathrm{mm})\end{array}$ \\
\hline $\begin{array}{c}{[38]-1 \mathrm{M}} \\
(\mathrm{d}, \mathrm{c}, \mathrm{a})\end{array}$ & SMA & yes $(1-2)$ & 2 & 12 & 49 & 14 & 2 & - \\
\hline [11] - (d,e) & SMA & no & 8 & 36 & 90 & 23 & 1 & 18 \\
\hline$[44]-(\mathrm{d}, \mathrm{a})$ & SMA & yes $(6-1)$ & 15 & 24 & 45 & 15.3 & 2 & 0.5 \\
\hline$[44]-1 \mathrm{M}(\mathrm{d}, \mathrm{a})$ & SMA & yes $(1-1)$ & 15 & 4 & 15 & 15.3 & 1 & 0.5 \\
\hline [47] - (a) & SMA & yes $(6-1)$ & 8 & 24 & 45 & 15.3 & 2 & - \\
\hline [47] - 1M (a) & SMA & yes $(1-1)$ & 8 & 4 & 15 & 15.3 & 1 & - \\
\hline [1] - (a) & SMA & yes $(1-1)$ & 4 & 9 & 12.5 & 41.2 & 1 & 0.37 \\
\hline [3] - (a) & $\begin{array}{l}\mathrm{SMA}+\mathrm{SDA} \\
\text { motor }\end{array}$ & yes $(2-1)$ & 1 & 2 & - & - & 2 & 0.05 \\
\hline$[15]-(d, e)$ & EAP (ICPF) & no & 0.8 & 10 & 42 & 13.5 & 1 & 2.85 \\
\hline$[40]-(\mathrm{d}, \mathrm{a})$ & EM motor & yes $(1-3)$ & 15 & 50 & 35 & 15 & 3 & 9 \\
\hline$[40]-1 \mathrm{M}(\mathrm{d}, \mathrm{a})$ & EM motor & yes $(1-1)$ & 8.5 & 21 & 105 & 10 & 1 & 6.5 \\
\hline$[33]-(\mathrm{d}, \mathrm{e}, \mathrm{a})$ & EM motor & yes $(1-3)$ & 7 & 25 & 15 & 10 & 3 & 1.05 \\
\hline$[46]-(\mathrm{d}, \mathrm{a})$ & EM motor & yes $(5-1)$ & 10 & 110 & 90 & 10.5 & 5 & 5.6 \\
\hline$[46]-1 \mathrm{M}(\mathrm{d}, \mathrm{a})$ & EM motor & yes $(2-1)$ & 10 & 36 & 90 & 10.5 & 2 & 5.6 \\
\hline [39] - (d) & Ultrasonic motor & no & 10 & 31.8 & 120 & 17 & 3 & 7 \\
\hline$[23,24]-(d, a)$ & Piezoelectric & yes $(5-2)$ & 10 & 250 & 345 & 72.7 & 10 & - \\
\hline $\begin{array}{l}{[23,24]-1 \mathrm{M}} \\
\quad(\mathrm{d}, \mathrm{a})\end{array}$ & Piezoelectric & yes $(1-2)$ & 10 & 50 & 69 & 72.7 & 2 & - \\
\hline
\end{tabular}

and Storz are producing endoscopes with classical distal bending and imaging capabilities, at the tip of a long flexible body, but adding one or several working channels in order to convey instruments and orientate them at their base. For example, Olympus produces endoscopes such as the GIF-Q180 ${ }^{\circledR}$ gastroscope [35] with an external diameter of $8.8 \mathrm{~mm}$ capable of bending in any direction with a maximum angle of $210^{\circ}$ in a preferential one, and providing a single working channel with an inside diameter of $2.8 \mathrm{~mm}$, or the CF-2T160 $\mathrm{I} / \mathrm{L}^{\circledR}$ colonoscope [34] with two working channels of 3.7 and $3.2 \mathrm{~mm}$ inside diameters for an external one of $13.8 \mathrm{~mm}$ (Fig. 1(a)). CambridgeEndo [29] developed the Autonomy ${ }^{\mathrm{TM}}$ Laparo-Angle ${ }^{\mathrm{TM}}$ Instruments integrating an articulation with $90^{\circ}$ bending capabilities in any direction, and an Axial Rotation Knob allowing to easily axially rotate the tool in any bending configuration, as seen in Fig. 1(b), useful capacity for needle driving while suturing for instance. Novare Surgical [20] is commercializing a manually driven laparoscopic instrument, called the Real Hand, providing orientation capacity of the distal part with a high dexterity in any direction but without axial rotation in a fixed bending configuration.

As mentioned previously, manually driven instruments limit the number of DOFs that can be simultaneously and intuitively controlled by the surgeon's hand through the handle. The number of controllable DOFs can be increased thanks to a robotic control of the articulations. This is the case with the Artisan ${ }^{\mathrm{TM}}$ Control Catheter [57] developed by Hansen Medical ${ }^{\circledR}$. This catheter, used to cure cardiac arrhythmias, is composed of three coaxial catheters of respectively 4.7,3.8 and 2.8 mm external diameters and develops in total eight intracorporal DOFs. An inner catheter can be translated out of its surrounding one and axially rotated, and each catheter has independent bending capabilities as seen in Fig. 2.

Another system commercialized for a clinical use and controlled by a robot for a more intuitive control is the second generation of the Endowrist ${ }^{\circledR}$ [8] from Intuitive Surgical ${ }^{\circledR}$. This articulation of $5 \mathrm{~mm}$ external diameter contains four segments that enable two decoupled DOFs (two segments per DOF) for bending with $90^{\circ}$ amplitude in two preferential directions.

An interesting system, more dedicated to travelling along complicated 3D trajectories inside the body than controlling its distal part, is the CardioARM ${ }^{\mathrm{TM}}$ described by Ota et al. [37] and commercialized by Cardiorobotics, Inc. [61]. Its structure is composed of two concentric articulated tubes that can be independently placed in a rigid or limp mode. By alternatively 


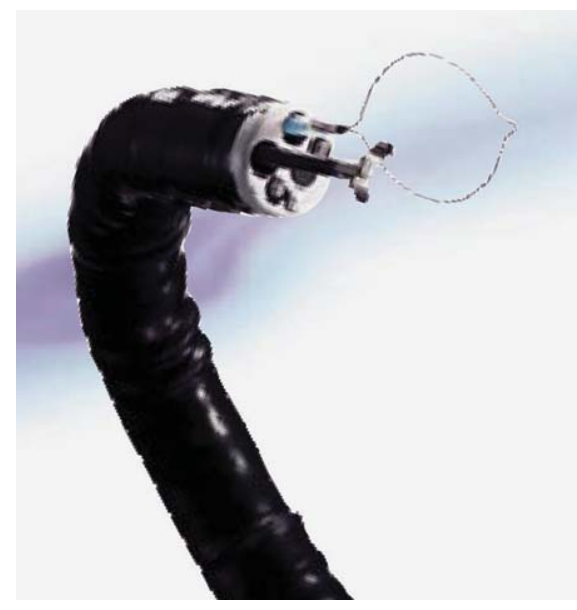

(a)

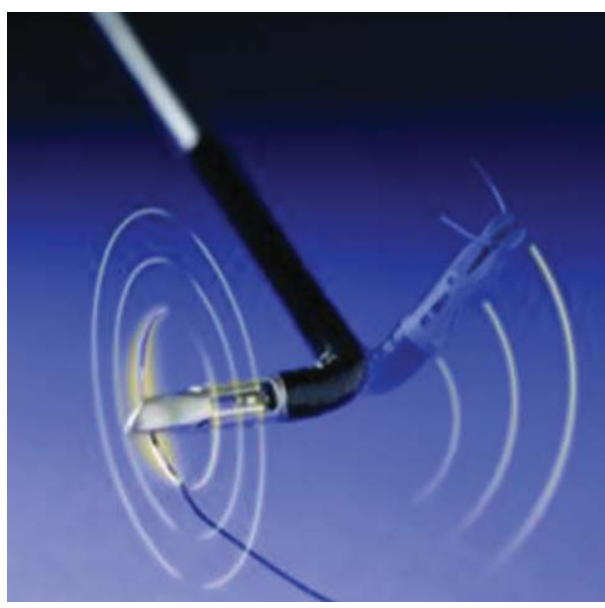

(b)

Fig. 1. (a) Olympus CF-2T160 ${ }^{\circledR}$ [34]. (b) Autonomy ${ }^{\mathrm{TM}}$ Laparo-Angle $^{\mathrm{TM}}$ instrument in a bent configuration [29].

Outer Guide

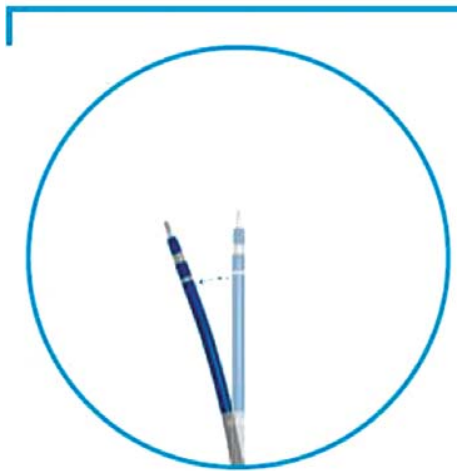

Proximal Bend $\operatorname{Max} 20^{\circ}$

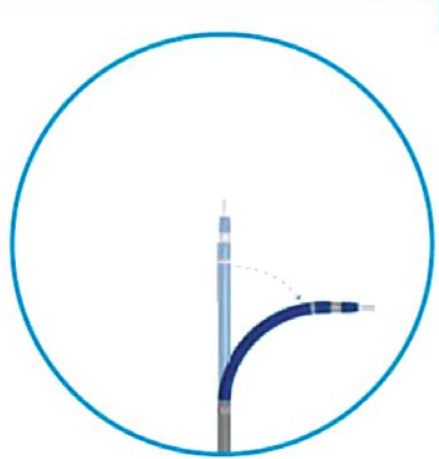

Distal Bend $\operatorname{Max} 90^{\circ}$
Inner Guide

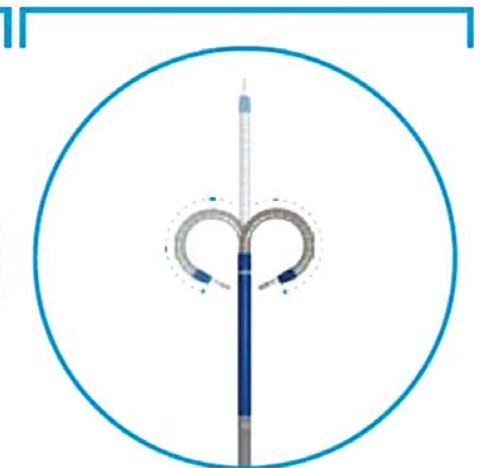

$270^{\circ}$ Deflection

in Any Direction

Fig. 2. Bending abilities of the ArtisanTM Control Catheter developed by Hansen Medical ${ }^{\circledR}$ [19].

placing the tubes in a limp/rigid mode and orientating the distal segment with $10^{\circ}$ amplitude thanks to the use of three cables, a step by step forward motion along a 3D complex trajectory is made possible (Fig. 3).

\subsection{Cable-driven articulations with pulleys and pivots joints}

A different articulation technology, using classical pivot joints, has been developed for the daVinci ${ }^{\circledR}$ robotic system by Intuitive Surgical ${ }^{\circledR}$ for the first gen- eration of its Endowrist ${ }^{\circledR}$ [31] with an $8 \mathrm{~mm}$ external diameter. Based on the PhD thesis of Madhani [30], this articulation uses three pairs of cables to drive two internal DOFs, allowing the bending of the tool in any direction with maximum amplitude of $90^{\circ}$. One of the DOF is obtained by an independent control of each jaw of the tool which results in an improved compactedness of the system with a length of approximately $30 \mathrm{~mm}$. Nevertheless, the fact that the two bending axes are not concurrent induces some unreachable positions and dead zones for the distal instrument. This problem 


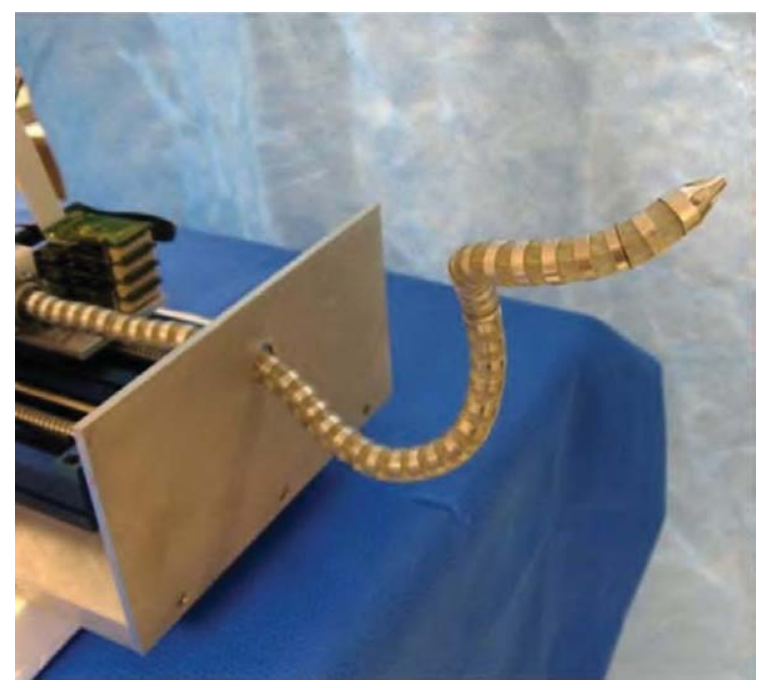

Fig. 3. 3D trajectory followed by the CardioARM ${ }^{\mathrm{TM}}$ developed by Cardiorobotics, Inc. [36] (C) [2008] IEEE).

can only be solved by reconfiguring the position of the entire robotic arm while taking care of all the collisions that may occur.

Endovia Medical, Inc. (recently acquired by Hansen Medical ${ }^{\circledR}$ ) has developed a compact system [56] based on the Endowrist ${ }^{\circledR}$ from Intuitive Surgical ${ }^{\circledR}$ but adding two intracorporal DOFs in order to limit the need of movements of the whole arm around the insertion point and allow a better access to areas difficult to reach inside the body. One bending up to $90^{\circ}$ and one axial rotation up to $180^{\circ}$ have been added to the Endowrist ${ }^{\circledR}$ of $8 \mathrm{~mm}$. Everything is being controlled from a small motorization box attached to the operating table, limiting the obstruction around the patient.

\subsection{Other technologies}

Many other technological solutions have been encountered in the commercialized medical systems. Medtronic ${ }^{\circledR}$ has developed the Cardioblate ${ }^{\circledR}$ Navigator $^{\mathrm{TM}}$ Tissue Dissector [53], a bipolar dissection device placed on the tip of a multi-link articulation that is controlled by a wheel facilitating a singlehand manipulation. The distal bending is possible only towards a single direction with $180^{\circ}$ amplitude and the transmission between the wheel and the bending tip is ensured by mechanical rods (Fig. 4(a, b)).

A more original device has been developed by Covidien Autosuture ${ }^{\circledR}$ (Covidien Autosuture 2010) for single incision surgery. The flexible articulation is prebent and kept straight as it is confined in a rigid shaft. Pushing the tool out of the shaft will free the articulation and allow the bending to occur to $80^{\circ}$ maximum amplitude as seen in Fig. 4(c). Therefore the translation of the tool and the bending of the articulation are coupled and depend on the external force applied to the tip which does not seem to be easily controllable.

Articulation technologies validated to be used in clinics are improving mainly through innovations regarding their structure, and enable to drive surgical tools along multiple DOFs. But that remains insufficient to reach some areas in the body, and then to position and manipulate the tools with a high dexterity after having reached these areas. Moreover they remain classical regarding their actuation technologies. Some other developments that have not been validated yet to be used in the medical environment are exploring different structural and actuation solutions and are presented in the following sections.

\section{Review of the articulation technologies \\ developed in $R \& D$ departments of companies and in academic research institutes}

As described previously, improvements are still needed for the realization of efficient articulations for minimally invasive medical applications and a large number of research groups and companies are working towards this goal. On the one hand, the solution of deported actuation is commonly adopted in order to avoid the main problem of miniaturization and integration of the actuation, as well as electrical compatibility for use inside the patient. In this case, innovation is generally concentrated on the structure and the transmission technology. On the other hand, several groups have chosen to deal with a local actuation. Many different actuation strategies and structural configurations have consequently been developed. Many of these developments, with local and deported actuation, are described in the following paragraphs.

\subsection{Deported actuation technologies}

\subsubsection{Rigid and flexible transmissions}

Mechanical transmission using rigid structures and developing at the most one intracorporal DOF for tool manipulation has been traditionally used for manually 


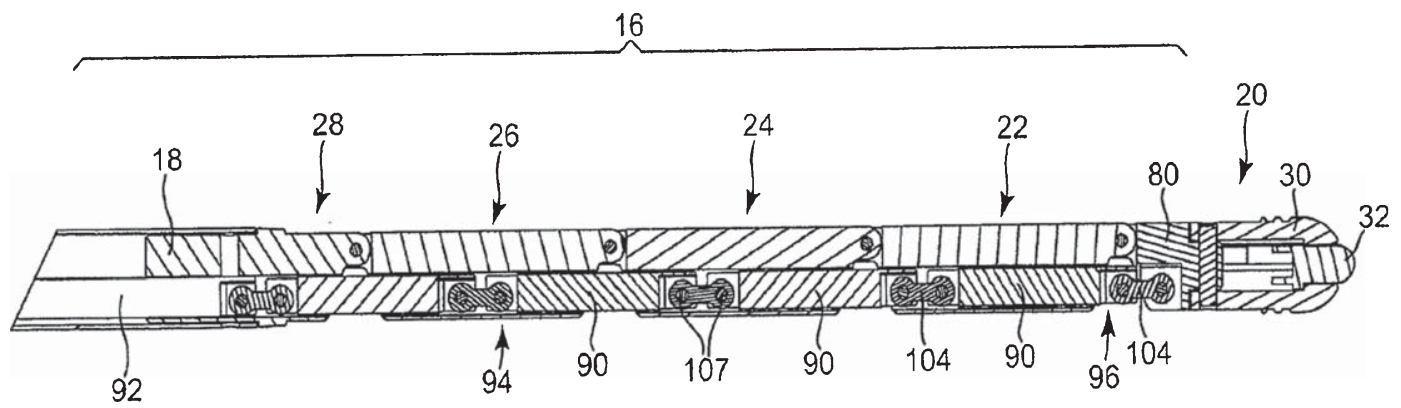

(a)

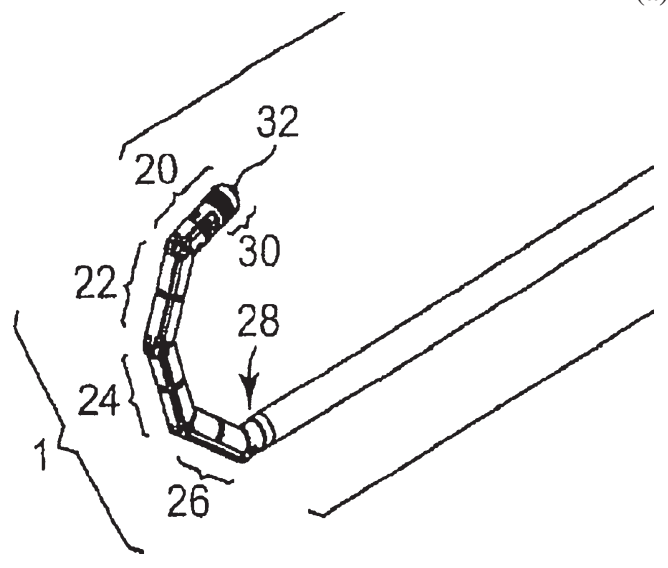

(b)

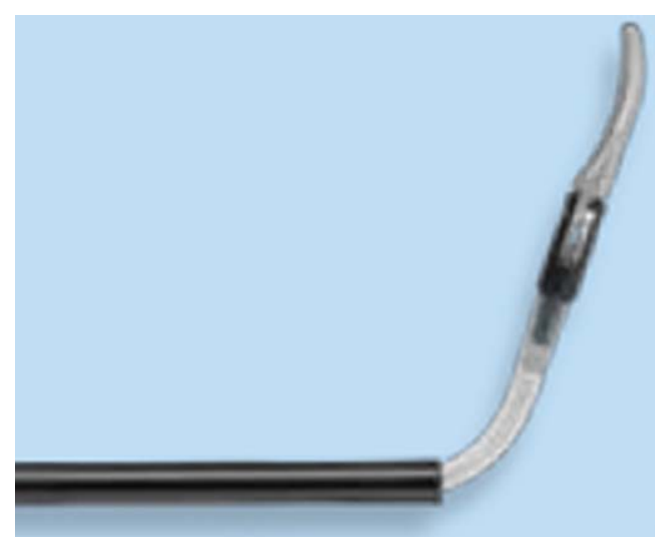

(c)

Fig. 4. (a, b). Cardioblate ${ }^{\circledR}$ Navigator ${ }^{\mathrm{TM}}$ Tissue Dissector mechanism [53]. (c) Covidien Autosuture ${ }^{\circledR}$ device (Covidien Autosuture 2010).

driven MIS instruments. It allows the transmission of large efforts but limits the possibility of controlling several DOFs at the distal part of a system with limited size. The implementation of a rigid transmission going through a snake like structure such as an endoscope or a catheter, necessary to reach the operation sites avoiding organs, seems rather difficult. Furthermore, this technology implies the manufacturing of complex mechanical parts of small dimensions, problems for assembling these parts, high frictions, and therefore it reduces the overall reliability of the system as well as its sterilizability.

Nevertheless, several articulations based on this technology have been developed, such as the Stewart platform based design developed by the ONERA [43] generating four DOFs (three DOFs of a ball joint and one translation) by the use of nine gimbals. As expected, the dimensions of this solution are important with an external diameter of $20 \mathrm{~mm}$. Intuitive Surgical ${ }^{\circledR}$ has also patented a similar structure [55] developing two DOFs. The best development achieved with this technology has been proposed by Yamashita et al. [59] with a transmission obtained by a slider linkages system going through a finger-like multi-joint structure that they have patented [12]. In this system, one DOF bending mechanism with $\pm 90^{\circ}$ amplitude is composed of three joints, one set of two linkages for drive and restraint, and a super-elastic shaft between the two proximal joints to ensure that the distal frame is rotating first (Fig. 5). A coupling of two of these mechanisms allows independent control of two DOFs. A miniaturized version [60] based on the same principle has been developed with an external diameter of $3.5 \mathrm{~mm}$, a length of about $15 \mathrm{~mm}$ and a working channel of $0.8 \mathrm{~mm}$ diameter.

Simaan et al. $[49,58]$ have developed an articulation with a transmission whose principle is a mixed concept between the rigid structures described above and classical transmission cables encountered in section 2.1. The structure is composed of four super-elastic NiTi tubes that ensure the rigidity of the structure, three of them ensure the actuation as well and are distributed 

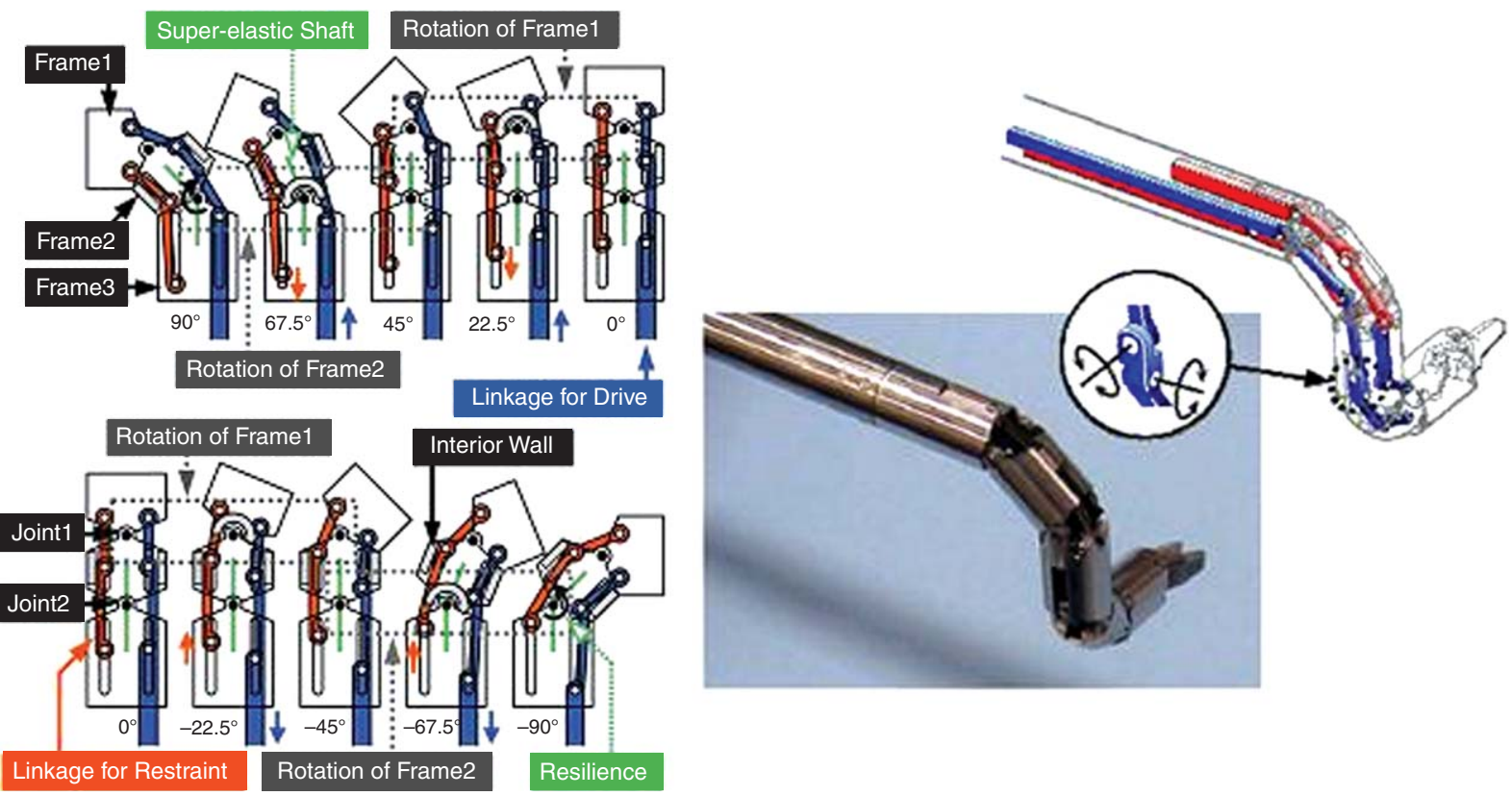

Fig. 5. Working principle scheme of the multi-slider linkage mechanism [59].

at $120^{\circ}$ around the cylindrical structure. Thanks to the axial stiffness of these tubes, actuation can be made by pulling or pushing them along the structure.

\subsubsection{Transmissions by mechanical cables}

Reflecting the most widespread technological orientation of the systems used in clinics, most of the articulations designed in academic research laboratories are relying on deported actuation with mechanical cables transmission. Again, a distinction can be made between architectures with their bending capabilities concentrated in a single joint and using mainly pivot joints, or structures with a more distributed bending capability.

\subsubsection{Cable-driven articulations with compact joints}

Some of these articulations are very similar to the Endowrist ${ }^{\circledR}$ developed by Intuitive Surgical ${ }^{\circledR}$ and using pivot joints for their joint architecture. The Robin Heart, developed by Podsedkowski et al. [42] introduces a supplementary DOF to the distal part for a maximum bending of $180^{\circ}$ or a radial translation as seen in Fig. 6(a). The system developed in the Institute of Robotics and Mechatronics of the DLR by Seibold et al. [48] considers a two DOFs wrist with pivot joints, similar to the first Endowrist ${ }^{\circledR}$ but with two concurrent axes. This geometry limits the presence of hard points and dead zones for a better dexterity. Generating large forces up to $20 \mathrm{~N}$, its dimensions $(10 \mathrm{~mm}$ diameter and about $15 \mathrm{~mm}$ length) are quite imposing.

Other systems are not using pivot joints to generate the bending movement from pulling cables, such as the Endoplatform developed by Cohn et al. [7] or the Hyper Finger developed by Ikuta et al. [21] from the Nagoya University in Japan. Ikuta et al. have augmented the number of intracorporal DOFs in a structure, whose first version was locally actuated by piezoelectric elements $[23,24]$ that have been replaced by an external actuation and a transmission by mechanical cables. Each DOF is actuated by a pair of cables for a total of 7 DOFs for a $3 \mathrm{~mm}$ diameter device (Fig. 6(b)).

\subsubsection{Cable-driven distributed articulations}

Articulations based on the compliance of a structure [4, $41]$ or an assembly of numerous joints of limited bending capabilities, have been developed in order to create highly flexible, but long, bending elements actuated by mechanical cables.

For instance, Breedveld et al. from the Delft University of Technology have realized several generations of bending structures actuated by cables. The last generation is a 2 DOFs $5 \mathrm{~mm}$ external diameter structure 

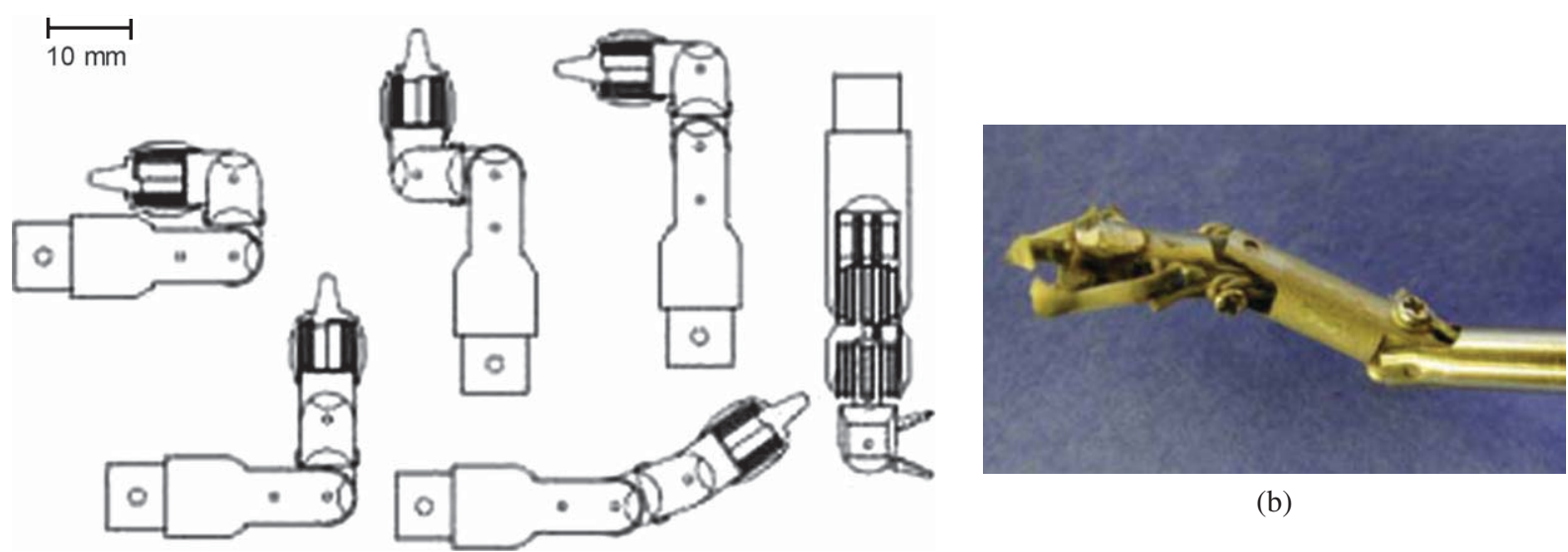

(b)

(a)

Fig. 6. (a) Manipulation abilities of the Robin Heart wrist [42]. (b) Tip of the Hyperfinger [26] (C) [2003] IEEE).

composed of two coaxial springs with actuating cables running between them axially as seen in Fig. 7(a). Van Meer et al. [54] have used the combination of joints with small bending capabilities (similar to the second generation of the Endowrist ${ }^{\circledR}$ [8]) for an articulation actuated by six pairs of cables. Compared to the Endowrist ${ }^{\circledR}$, each joint is able to bend along two DOFs, therefore improving the compactness of the articulation and the ability to bend in any direction with maximum amplitude. Based on the same architecture, Harada et al. [18] have realized a miniaturized articulation driven by 4 cables with an external diameter of $2.4 \mathrm{~mm}$ for a bending amplitude of $180^{\circ}$ (Fig. 7(b)). A comparable system has been developed by Novare ${ }^{\circledR}$ [10] but not yet commercialized. This architecture enables to control the bending of snake-like structures with numerous DOFs, each segment being oriented independently by a set of three cables as shown in Fig. 7(c). Therefore the cables controlling the distal segments are running through the proximal ones, probably limiting the number of implementable segments and the actuation force that can be applied on the distal segments, without affecting the orientation of the proximal ones.

\subsubsection{Fluidic actuation}

Fluidic actuation is considered as deported, as the actuator is in fact the compressor whose energy is transmitted by a fluid to articulations that can be named "fluidic actuators" and shares similar advantages and drawbacks. They have the advantages of generating large forces and displacements with good dynamics; furthermore they can be supplied by physiological saline. Nevertheless, they need large supply hoses for large pressure and their control is not easy. They can be separated into two categories, depending on how the movement is generated. In the first category, bending is coming from a pressure difference between chambers, and in the second category, bending is generated by a pressure change in a structure presenting anisotropic rigidity.

\subsubsection{Bending from internal chambers pressure difference}

One of the first fluidic actuators considered for medical applications was developed by Suzumori et al. [50]. It is called flexible microactuator (FMA) and consists of a silicone rubber cylinder presenting three longitudinal internal chambers. It is reinforced with nylon fibres in order to avoid radial expansion when pressurized, and generates bending in any direction by independently controlling the pressure in each chamber (Fig. 8(a)). When each chamber is equally pressurized, a translation is generated. The translation can be coupled with an axial rotation by inserting the nylon fibers in a spiral shape around the cylinder creating an anisotropic elasticity. Due to the simplicity of its structure, this articulation can be easily miniaturized. Chiba et al. [6] have described for Asashi Optical a similar articulation adding an SMA spring to improve the actuation.

An articulation based on pre-shaped balloons and active micro valve system has been developed by 

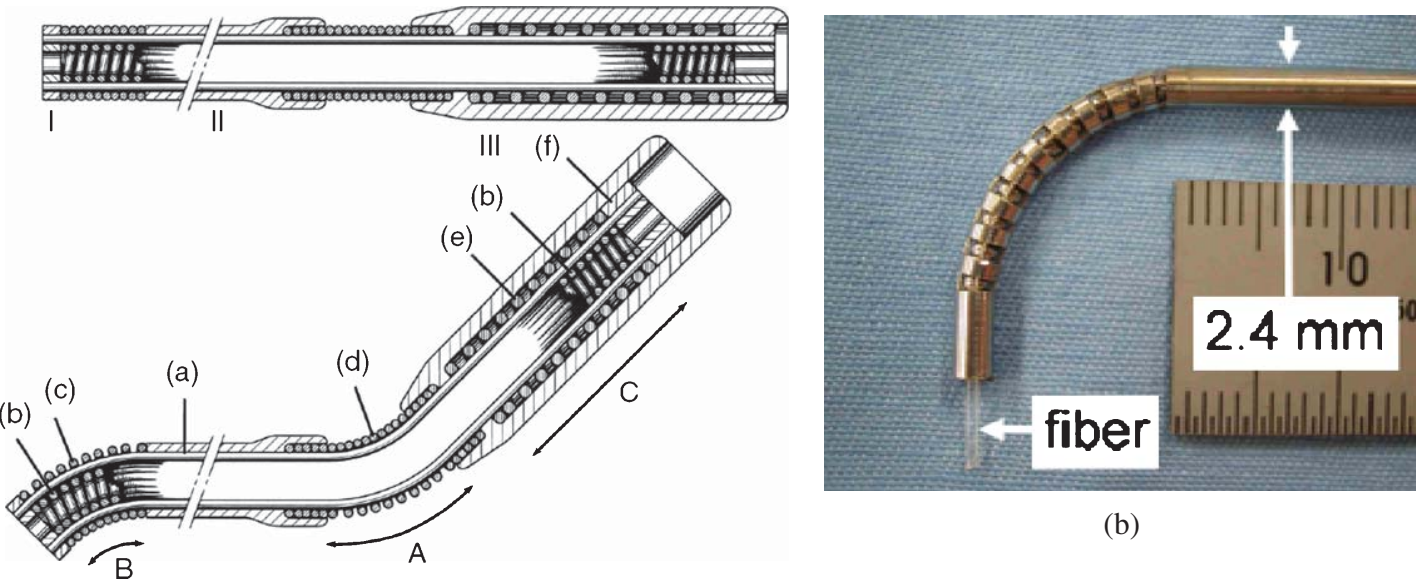

(b)

(a)

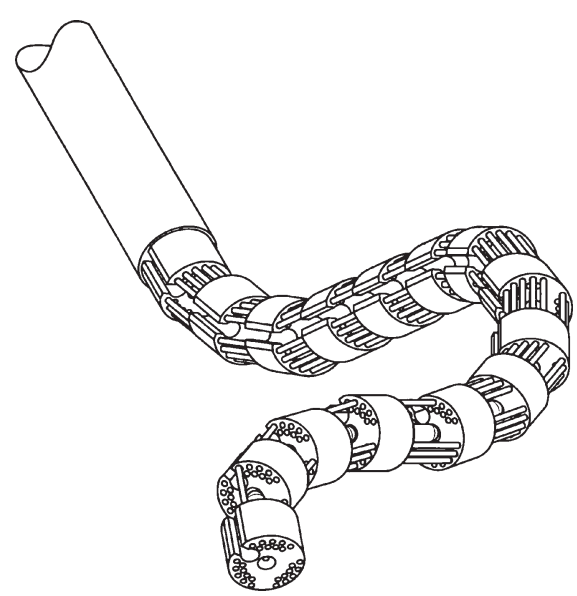

(c)

Fig. 7. (a) Flexible articulation developed by Breedveld et al. [4] (@ [2005] IEEE). (b) Articulation developed by Harada et al. [18] (@ [2007] IEEE). (c) Representation of the snake-like structure developed by Novare ${ }^{\circledR}[10]$.

Ruzzu et al. [45] in order to orientate a laser beam in blood vessels with low actuation pressures (Fig. 8(b)). The fluid is flowing through the balloons and the differential pressure to inflate them is controlled by the flow rate difference between inlet and outlet. The electrothermo-pneumatically actuated micro valves, normally open, control the inlet flow rate actively as they are closing. The maximum bending amplitude reachable when the balloons are inflated is $5^{\circ}$ for a $5 \mathrm{~mm}$ diameter and $4 \mathrm{~mm}$ length.

Articulations based on three bellows radially distributed at $120^{\circ}$ apart were developed by Thomann et al. [52], Kim et al. [27] and Bailly et al. [2] seen in Fig. 8(c). By adjusting the pressure in each bellow independently, they can generate displacements along three
DOFs. Another possibility proposed by Peirs et al. [40], is to implement 3 hydraulic jacks to actuate a Stewart platform along 3 DOFs. This solution generates large forces and bending with $35^{\circ}$ amplitude.

\subsubsection{Bending due to anisotropic rigidity}

An internal pressure change can generate the bending of a structure if it presents an anisotropic rigidity, influencing the repartition of the fluid volume. Such principle has been used by Ikuta et al. [22] to develop a catheter composed of anisotropic bellows, whose only one side can extend resulting in the bending in one direction. The bellows are linked in order to form a catheter with several DOFs. Thanks to pressure controlled valves set to open at different pressures for 


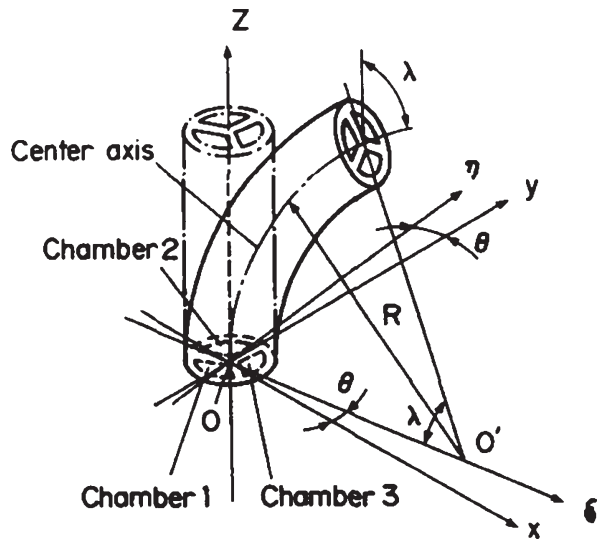

(a)

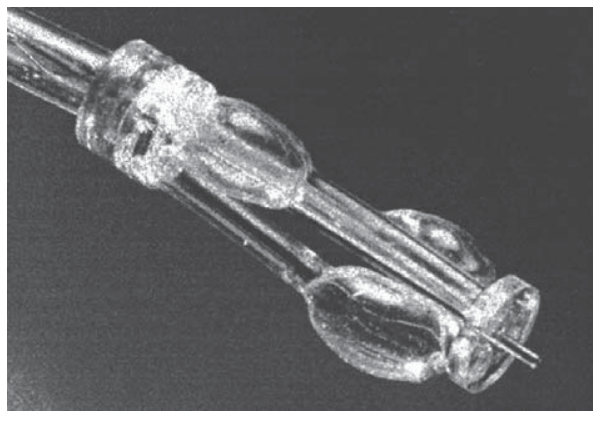

(b)

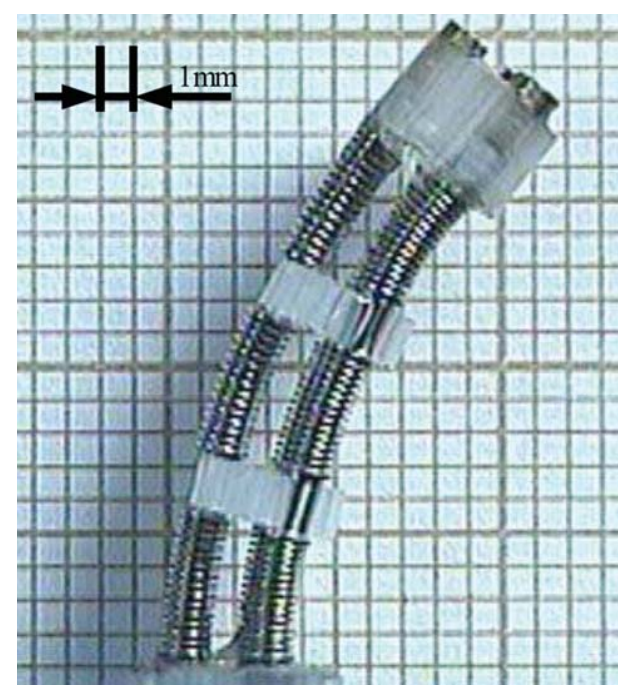

(c)

Fig. 8. (a) Fluidic Micro Actuator [50] (C) [1991] IEEE). (b) Balloon activated laser positioning system [45]. (c) Bellows based articulation [2] (C) [2005] IEEE).

each bellow, the system needs a single fluid supply and pressure control. This implies that the bellows can only be sequentially controlled and not independently. Thanks to the use of micro stereophotolithography fabrication process, highly miniaturized valves have been built, reducing the diameter of the catheter to $1.5 \mathrm{~mm}$ (Fig. 9(a)). Haga et al. [16] have described a $0.94 \mathrm{~mm}$ diameter structure that can develop a bending of $160^{\circ}$ along one DOF by suction of the filling fluid. The structure is made of a laser micromachined tube of NiTi with a silicone rubber tube to ensure sealing (Fig. 9(b)).

Despite the advantages of choosing a deported actuation (which reduces the actuation dimensional constraints), these structures present drawbacks due to a bulky transmission that, in case of solid mechanical transmissions, generates important frictions as well. These frictions can be compensated by increasing the actuators performances, but they are difficult to measure accurately, limiting fine movements capabilities and small resulting efforts. Mechanical cables, pivot joints, gears or pipes used for mechanical and fluidic transmission can not be miniaturized extensively limiting the size reduction of the whole mechanism. Integrated actuation is an alternative to solve these problems, but developments are needed in order to achieve the forces and dynamic requirements and to overcome the integration problems. Attempts will be described in the next chapter. 


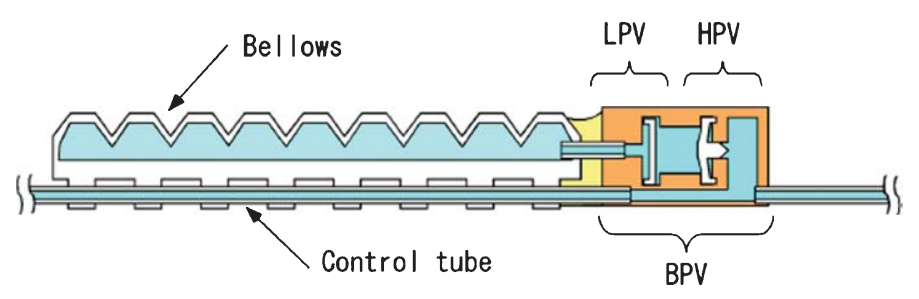

(a)

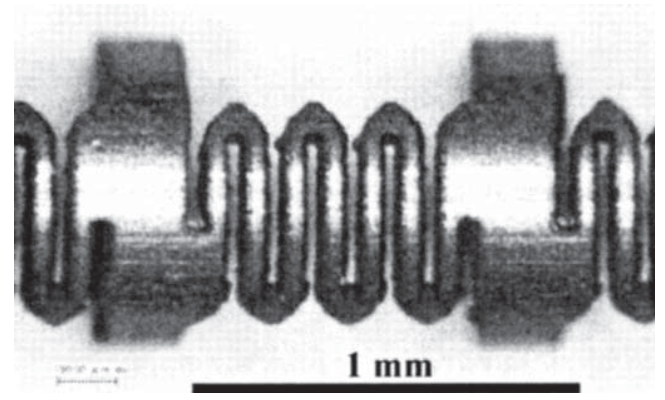

(b)

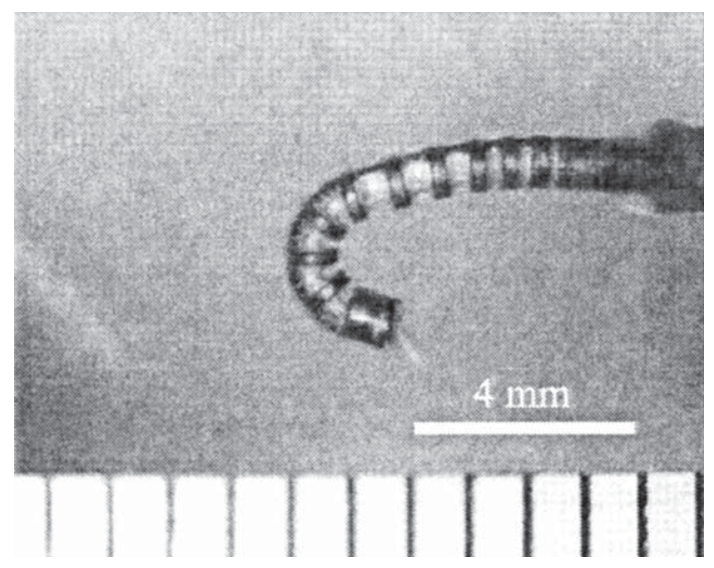

(c)

Fig. 9. (a) Pressure controlled valve and its associated bellow [22] (@ [2003] IEEE). (b, c) One DOF articulation developed by Haga et al. [16] (C) [2005] IEEE).

\subsection{Integrated actuation technologies}

Building up an articulated structure with a local actuation presents the advantages of reducing the energy loss in the transmission chain and, in the specific case of a reversible actuation technology, the possibility of obtaining a force or displacement integrated measurement. Furthermore, a local actuation allows the conception of modular mechanisms with high mobility and a kinematics as complex as needed. Nevertheless the remaining difficulty is to combine the actuation miniaturization with the generation of large forces and displacements compatible with the application domain. The development of new articulation structure to amplify the performances of the actuation technology is an issue to consider as well. Several devices have been developed and are described in the following classified by actuation technologies.

\subsubsection{SMA actuators}

Shape Memory Alloys (SMA) have got advantages that explain their wide use in the biomedical field. They present a large mechanical energy density to produce large forces and displacements with limited volume, they can tolerate deformations up to 6 to $8 \%$ and $\mathrm{NiTi}$ alloys are biocompatible. As drawbacks, their actuation temperature can be high, they are not suitable for applications requiring high dynamics, their highly non-linear behavior makes them difficult to control precisely, and they develop forces in only one direction (so they have to be used in an antagonistic way or to be coupled with a return device). The miniaturization of the active parts in SMA can reduce the time response down to few tens of milliseconds, but reduces the forces generated as well. SMA have already been used in devices designed for use in the medical domain but mainly dedicated to simple procedures that do not 
require movements with high dynamics and dexterity (such as the orientation of the extremity of catheters or endoscopes to facilitate advance in natural pathways or the orientation of the camera during exploration procedures). They could also be used for simple tasks such as coming into contact with tissues to burn them, realize an injection or a biopsy.

Different mechanical architectures can be found in the articulations actuated by SMA that have been developed. Most of them present an architecture similar to the articulations actuated by cables, except that pulling the cables is replaced by contracting SMA wires (or springs) that are equally distributed around the main axis of the structure [5, 25, 51]. Haga et al. [17] have developed three articulations of this type with different structural configurations. One has its rigidity ensured by polymer flexible links, another by an inner flexible tube as the backbone of the articulation, and the last one by an outer hose. They comprise flexible segments with limited bending amplitude which can be combined and actuated independently increasing the number of DOFs. Another version developed by Park et al. [38] comprises CMOS integrated circuits for the independent control of each segment with only three electric power supply wires as seen in Fig. 10(a).

Inspired from Grant et al. [14], Dario et al. [11] from the University of Pisa have developed a slightly different articulation composed of disks connected by two continuous SMA antagonistic wires as seen in Fig. 10(b). This arrangement amplifies the steering abilities of the structure for a given deformation of the SMA wires.
Reynaerts et al. [44] have introduced a system composed of 1 DOF bending modules that can be combined to increase the bending amplitude or form a full articulation with two DOFs. Each module is composed of two identical parts rotated by $180^{\circ}$ from each other, placed on top of each other and connected by two super-elastic joints and two actuation SMA strips as shown in Fig. 11(a). The same modular configuration has been used by Szewczyk et al. [47] with different module architecture. Each module is composed of two iron rings joint together by interlocking as seen in Fig. 11(b). Two antagonist SMA spring actuators are inserted between the rings in order to modify their relative orientation.

Another ring-shaped modular based system has been developed by Abadie et al. [1] that consists in antagonistic NiTi blades that bend in opposite direction when heated. Thermoelectric junctions $\mathrm{P}$ or N-doped are placed between the structural rings and the SMA blades, in order to generate a reversible Peltier thermoelectric effect for heat production or absorption. As a blade is heated up, the other one is cooled down, accelerating the reverse transformation of SMA and therefore the overall dynamic of the system.

Bourbon et al. [3] have described a catheter with $1 \mathrm{~mm}$ external diameter that can generate a bending along one DOF thanks to an SMA actuation but having the particularity of developing an axial rotation thanks to a rotative motor based on electrostatic Scratch Drive Actuator (SDA). The ring motor has a diameter of $1 \mathrm{~mm}$ for $2 \mathrm{~mm}$ length and integrates 1430 SDA generating torque of $50 \mu \mathrm{Nm}$ with speed from

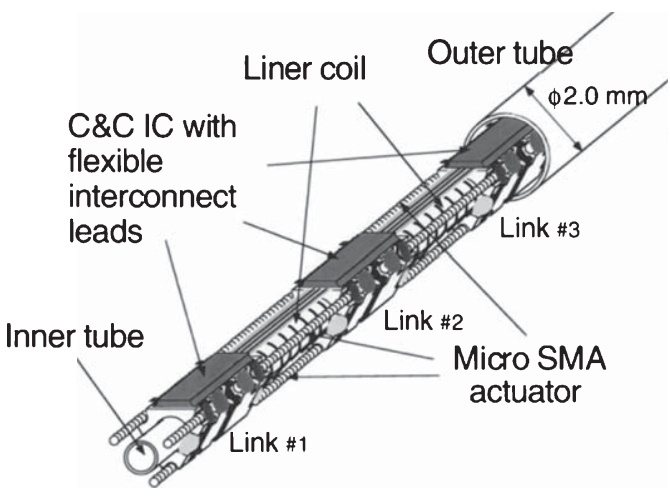

(a)

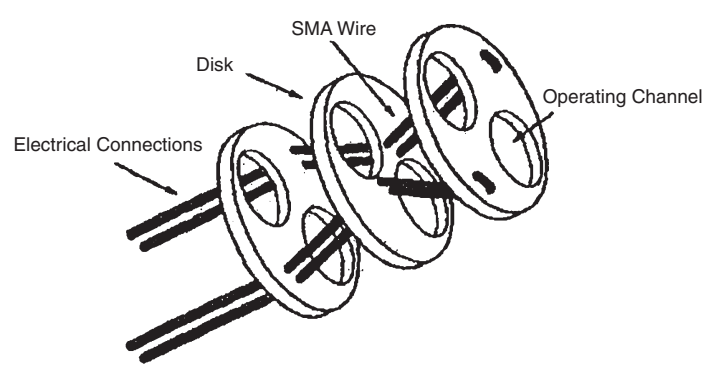

(b)

Fig. 10. (a) Modular structure developed by Park et al. [38] (๑ [1999] IEEE). (b) SMA wire implementation in the articulation developed by Dario et al. [11] (@ [1997] IEEE). 


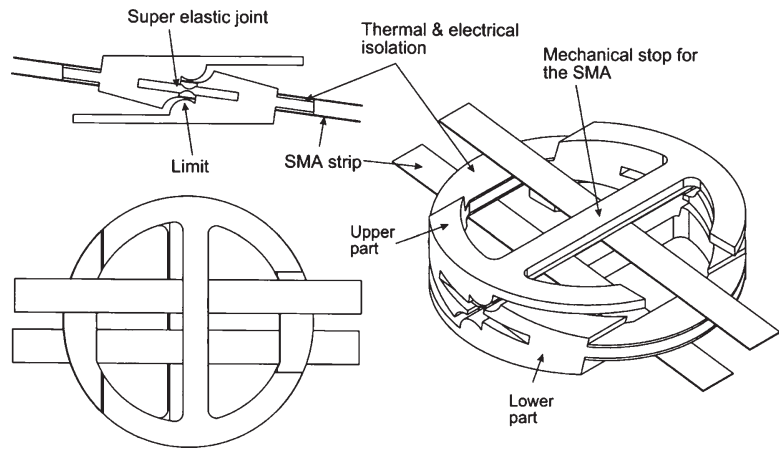

(a)

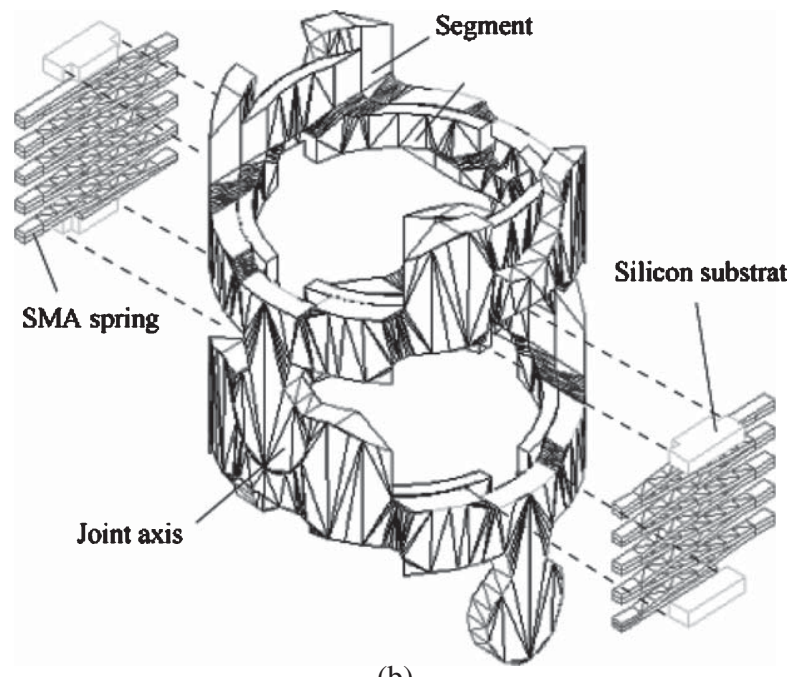

(b)

Fig. 11. (a) Bending modules developed by Reynaerts et al. [44]. (b) Bending modules developed by Szewczyk et al. [47].
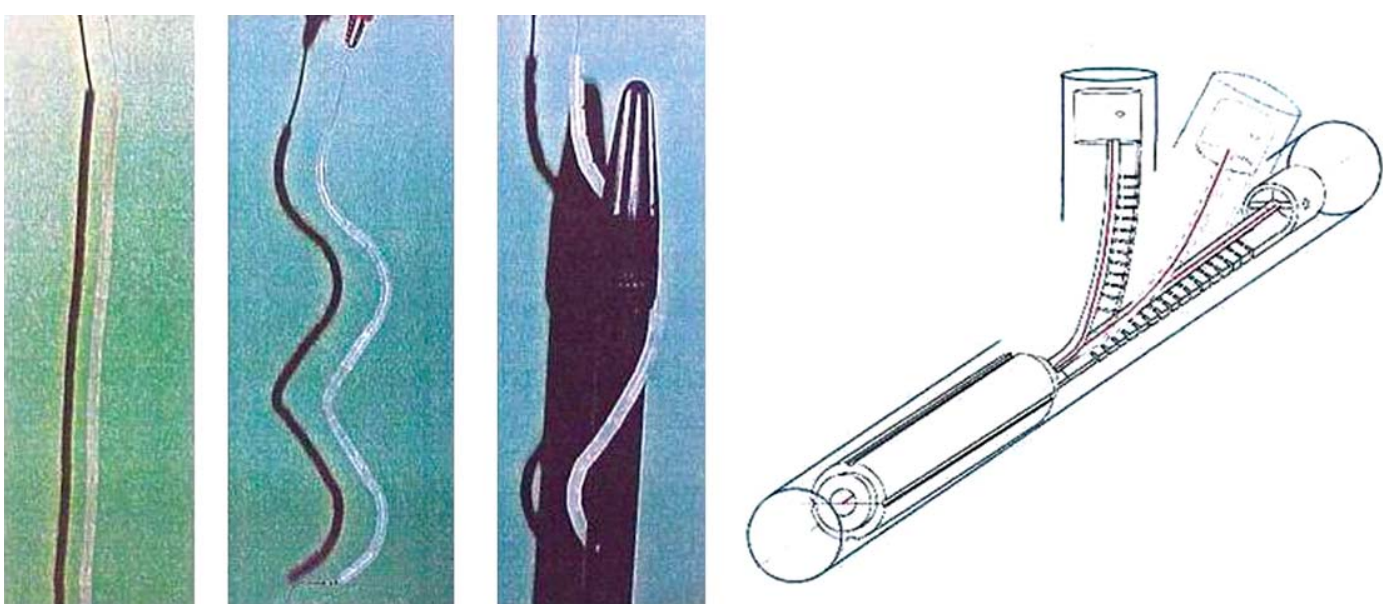

Fig. 12. Active micro-catheter developed by Bourbon et al. [3] composed of two DOFs modules.

$10^{-3}$ to $300 \mathrm{rpm}$. Several module generating one bending and one axial rotation can be combined as seen in Fig. 12.

\subsubsection{Electro active polymer actuators}

Electroactive polymers (EAP) represent a whole family of active polymeric materials that can be organized in different groups, depending on the physical mechanism that takes place during their actuation. Two main groups can be distinguished, electronic EAP and ionic EAP. Electronic EAP do not seem to be suitable for medical applications, mainly because of the high actuation voltage needed (more than $150 \mathrm{~V} / \mu \mathrm{m}$ ) even if they can generate large forces with good dynamics. On the opposite, ionic EAP can be actuated with few volts but they need to be placed in an aqueous environment and their actuation principle based on molecular diffusion is slow and does not generate large forces. Nevertheless, some devices using these actuators have been proposed.

Guo et al. have developed [15] a catheter using Ionic Conducting Polymer Film (ICPF) being from the most efficient ionic EAPs. The catheter is composed of a polymeric cantilever $200 \mu \mathrm{m}$ thick, $800 \mu \mathrm{m}$ large and $10 \mathrm{~mm}$ long and covered by $3 \mu \mathrm{m}$ thick platinum electrodes. The cantilever can bend along one DOF to $42^{\circ}$ amplitude within $0.2 \mathrm{~s}$. The use of EAP for flexible 


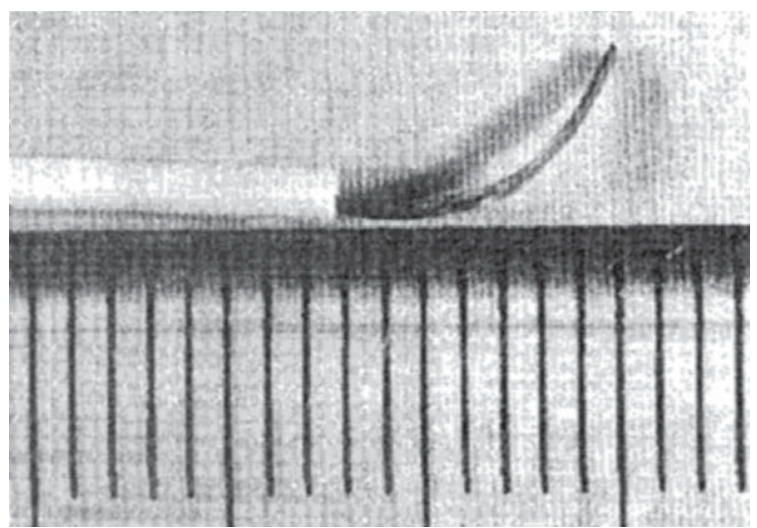

Fig. 13. EAP actuated cantilever [15].

catheters or endoscopes actuation has been patented by Couvillon et al. [9] from SciMed Life systems Inc. (Fig. 13).

\subsubsection{Electromagnetic motors}

Electromagnetic (EM) motors which have evolved, are able to generate relatively high torques with small dimensions and are easy to control. Nevertheless, they generate only one rotational movement and need to be coupled in order to obtain a structure with several DOFs. Furthermore they generate electromagnetic fields that can perturb neighbouring operating room devices.

Peirs et al. [40], as an improvement from their fluidic jack actuated Stewart platform, have integrated brushless motors to actuate this platform, and so did Merlet [33]. They finally developed one DOF module actuated by a brushless motor in order to reduce the diameter of the structure. These modules can be coupled in order to increase the number of DOFs to the desired number. Sallé et al. [46] have developed the same type of one DOF modules adding a module generating an axial rotation. They described a combination of 5 modules with a kinematics enabling a coronary artery bypass grafting.

\subsubsection{Ultrasonic motors using piezoelectricity}

Piezoelectric materials have been used to build ultrasonic motors that present many advantages compared to electromagnetic motors. In the case of a simple rotative motor with one DOF, the rotation is obtained by generating a propagating surface acoustic wave from the combination of two stationary surface acoustic waves with $\pi / 2$ dephasing. Each point on the surface of the stator in contact with the rotor is thus describing an elliptical trajectory which drives the rotor. As opposed to electromagnetic motors, ultrasonic motors can generate linear motions together with combinations of several rotations; they present a higher power density, a higher torque for a lower speed, they are blocked when not actuated (therefore not consuming power) and there is a relative flexibility in their shape even if great care should be taken in the vibration characteristics of their structure. Nevertheless, they present limited life time due to friction between the rotor and stator, their control is complex, the supply voltage is high and they present a low energetic efficiency.

Park et al. [39] have developed a ball joint articulation that can orientate surgical tools along 3 DOFs. The motor is composed of a cylindrical stator and a spherical rotor. Three vibration modes can be generated in the stator that can be combined to get 3 DOFs of the rotor.

\section{Performances comparison and discussion}

As announced previously, this literature review has the objective to present the different technologies of articulations developed for minimally invasive medical applications. This paragraph deals with a more accurate comparison of their mechanical performances. This will enable us to point out the advantages and drawbacks of each actuation technology coupled with structural choices regarding the medical and surgical application domains of interest.

As stated in the introduction of this paper, the comparison is based on criteria of main importance for the evaluation of the performances of such systems. The chosen criteria are the size of the articulation (i.e. external diameter and length), the number of DOFs, the maximum bending amplitude, the radius of curvature and the torque generated. The comparison could not be based on other criteria since they are rarely mentioned by the authors.

\subsection{Definitions}

1. An articulation refers here to the moving parts of the whole structure, including the actuators, when they are locally integrated, and the transmission parts directly in contact with the structural moving parts. We exclude the transmission parts such as cables, or rods, which are solely travelling 
through the structure from the actuators when deported. For articulations composed of several elementary identical modules that can be combined, a single module will be evaluated and referred as an articulation; the possibility of combining the modules (i.e. articulations) is notified by the note (a) in Table 1 .

2. The dimensions of an articulation, assuming the previous definition, are its maximum external section diameter and its length. In case of a multiDOFs articulation, the given value of the length will include all the DOFs mentioned. If structural passive parts, not necessary for the generation of movements, over-evaluate the length, an objective estimation of the dimension necessary to generate all the DOFs (without the passive parts) is given and notified by note (b) in Table 1 . When length has been estimated by scaling the given value of the diameter from pictures or drawings, this is notified by legend (c) in Table 1.

3. The bending amplitude given is the maximum bending angle reachable by the most efficient bending DOF of the articulation.

4. The radius of curvature corresponds to the one of the most effective bending DOF. When not given by the authors, the radius of curvature is estimated (note (d) in Table 1) from the length of the part of the articulation driving this DOF and its bending amplitude.

5. The torque given is the torque generated by the most efficient bending DOF. When information on the torque generated is not found in the literature, we deduce the torque from the force generated at the tip of the articulation and the radius of curvature (considered as the lever arm) (note (e) in Table 1).

\subsection{Reference criteria}

In order to have a reference to more easily compare and evaluate the state-of-the-art devices performances, values of these different criteria are chosen corresponding to either requirements or objectives for enhanced minimally invasive surgery devices development. Thus, the optimal structure would be modular in order to adapt its performances to the procedure to perform. The goal for the external diameter is set to $3 \mathrm{~mm}$, which corresponds to the internal size of some of the smallest trocars currently available. The number of DOFs interesting for one articulation is set to four, two bending DOFs, one axial rotation and one translation. The maximum bending angle required is set to $90^{\circ}$ in any direction, a value that seems reasonable to perform many tasks, as far as the radius of curvature is small and elementary articulations can be combined together. The radius of curvature is set to be as small as possible, in order to orientate the tools in a very confined space, taking into account the given diameter and bending angle requirements. Thus, the radius of curvature requirement is set to $1.5 \mathrm{~mm}$. From the radius of curvature and the bending angle, the required length for generating the two bending DOFs is deduced to be set to $2.5 \mathrm{~mm}$ in order to obtain the most compact structure possible. The same volume is considered for the two DOFs left, i.e. the axial rotation and the translation, leading to a total length of $5 \mathrm{~mm}$ for the whole elementary articulation. These targeted values, as well as the ones characterizing the devices from literature quoted in the present article, can be found in Table 1.

\subsection{Normalized criteria}

To facilitate the comparative study of the technologies, a graphical representation is proposed here. For this graph, the majority of the criteria are used and normalized around the objective performances previously described. Thus, a criterion reaching the objective performance will be quoted as 1 . An articulation is more efficient regarding any criterion as the normalized criterion value is increasing. The module with the worst efficiency regarding one criterion will be quoted 0 .

For a criterion that increases with efficiency (i.e. bending angle and number of DOFs) the normalized value $\mathrm{C}_{\mathrm{i} \text {-norm }}$ for this criterion is calculated from the Equation (1).

$C_{i-\text { norm }}=\frac{C_{i}-C_{\text {min }}}{R-C_{\min }}= \begin{cases}0 & \text { for } C_{i}=C_{\text {min }} \\ 1 & \text { for } C_{i}=R\end{cases}$

where $R$ is the required value of the criterion, $C_{i}$ the value of the criterion for the articulation $i$, and $C_{\min }$ is the minimum value of this criterion among all referenced state-of-the-art modules.

For a criterion that decreases with efficiency (i.e. diameter, length and radius of curvature), the normalized value $\mathrm{C}_{\mathrm{i} \text {-norm }}$ for this criterion is calculated from the Equation (2). 
$C_{i-\text { norm }}=1-\frac{C_{i}-R}{C_{\max }-R} \begin{cases}0 & \text { for } C_{i}=C_{\max } \\ 1 & \text { for } C_{i}=R\end{cases}$

where $\mathrm{C}_{\max }$ is the maximum value for this criteria among all referenced state-of-the-art modules.

Finally, in order to build a readable graph, one best system is selected among each technology. This best system is defined using domination rules as follows. The dimensions are considered the main criteria, especially the diameter, directly related to the size of the incisions. Then, the maximum bending angle and the number of DOFs are considered; the radius of curvature is considered at last. The number of DOFs and the maximum bending angle can be considered the less predominant criteria in the case of an articulation composed of modules which can be optimally combined to improve the performances. Some technologies are used in systems that are either commercialized or not validated for clinical use; in this case one system per category will be chosen to be represented in the graph. Figure 14 shows the graph obtained using all these considerations.

\subsection{Discussion}

From Table 1 and Fig. 14 we can stand some general considerations on the advantages and drawbacks of the different technologies represented.

As expected, systems with deported actuation and solid mechanical transmission have the main advantage of generating large forces about one order of magnitude above the solutions with integrated actuations. Their main drawback is due to the size and complexity of the mechanical transmission, which reduces the number of DOFs for a given dimension, due to assembly problems and resulting reduced reliability, and limits their modularity potential. Solutions with rigid transmission are the most affected by these limitations, even if the group from the University of Tokyo $[12,59,60]$ have come up with a strongly miniaturized solution. One exception can be found with the CardioARM $^{\mathrm{TM}}[37,61]$ as it can be considered as partially modular, since the number of segments can be largely increased in order to follow more complicated trajectories, but only one segment is controllable at a time. Nevertheless, they are able to generate large

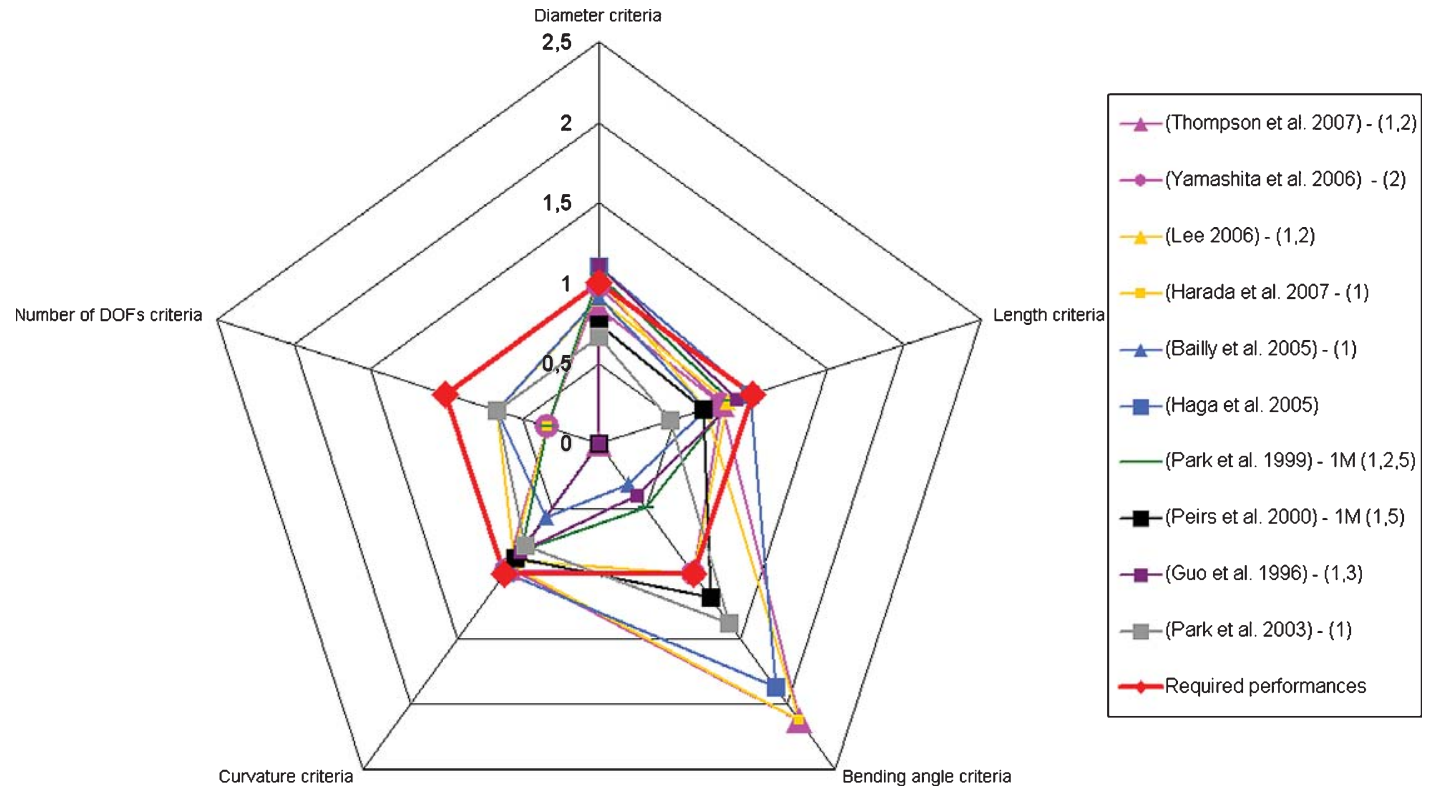

Fig. 14. Performances comparison of micro-articulations developed for medical applications based on different actuation technologies, using normalized criteria (see $\S 4.3$ ). (1) Refers to an estimated radius of curvature; (2) refers to an estimated length; (3) refers to an estimated torque; (4) refers to an estimated length after removing the passive structural parts; (5) refers to modular systems. Refers to rigid or flexible transmission, $\square$ to mechanical cables transmission, $\square$ to fluidic transmission, $\square$ to SMA actuation, $\square$ to electromagnetic motors actuation, $\square$ to EAP actuation, $\square$ to ultrasonic motor actuation and $\square$ to the reference performances. 
bending angles with dimensions comparable to the other systems.

Fluid actuated systems can produce non negligible forces, but suffer from the encumbrance of the supply circuit, limiting the possibility of local pressure or local volume control and therefore limiting their modularity as well. But Ikuta et al. [22] have managed to develop a highly miniaturized partially modular fluidic articulation. Fluidic systems based on anisotropic flexibility of the structure, such as the one developed by Haga et al. [16], generate few DOFs but they can be very compact and develop large bending angles. Fluidic systems based on the pressure difference between separated chambers, such as the system from Bailly et al. [2], develop more DOFs but smaller bending angles and they are larger.

Comparatively, systems with integrated actuation have the strong advantage of being potentially modular. Systems actuated by SMA elements, which is a rather mature actuation technology, can be built by assembling fully independent modules such as the system developed by Park et al. [38] and can therefore produce several DOFs in a compact structure. Their drawbacks are the long response time of the actuator due to thermal effect, the limited displacement generated inducing a limited bending angle, and the fact that forces can generally be generated in only one direction.

Systems actuated by electromagnetic motors can be modular and their main advantages are that their control laws are well established and they present good dynamics. The main drawbacks are the encumbrance of the motor that can generate only one DOF and the need for a space consuming mechanical reduction stage (gear box) in order to increase the output torque and to reduce the speed of the motor. On the other hand, the movement amplitude of the generated DOF can be large as it is only limited by the structure.

The system developed by Guo et al. [15] and actuated by ionic EAP is interesting in terms of size. Although this technology needs more developments to be further implemented in a surgical system to be used in clinics, it will probably not show force and dynamics characteristics compatible with surgical gestures.

It can be seen that ultrasonic motors can produce several DOFs and could be coupled in order to increase this number, but the motors developed so far present dimensions that are too large for this application. This is due partially to the fact that the torques generated depend on the preload between the rotor and the stator, which imposes that the structure must be robust enough to support these constraints.

From this paragraph it can be concluded that there exist many different candidate solutions to build active articulations with performances compatible with minimally invasive medical applications. The dimension of these articulations is, together with force and dynamics properties, one of the most important criteria to be evaluated, and it can be seen that some technologies do not present large perspectives towards miniaturization. This is the case, in the present state of their development, for articulations with deported actuations, or using electromagnetic motors or piezoelectrically based ultrasonic actuation. Fluidic actuation has a strong potential for miniaturization but still depends on a technological solution to locally and independently control the pressure and volume of the fluid in a large number of channels or chambers from a unique fluid supply tubing.

The other solutions are all sharing advantages and drawbacks. Some of them have not been studied for a long time and present a great potential for improving their implementation in efficient systems, such as the ionic and electronic EAP actuators. Others, such as the SMA, have been extensively used for this kind of applications, but research groups could still bring improvements (such as in the device from Abadie et al. [1]) in order to improve the dynamics of the actuation, one of the main drawbacks of these actuators.

\section{Conclusion}

This paper gives one of the largest overview of the articulations developed for devices to be used in minimally invasive medical applications and shows the variety of actuation technologies that have been explored to date. These technologies have been compared through the related mechanical performances that can be expected when using them. A large database of the performances reached by these systems and a graphical representation of the ones of the most efficient devices, classified by actuation technology, are given and compared with surgery field reference performances. This comparison shows that this application domain still needs the development of more efficient systems. The present analysis can therefore serve as a basis to the designer, in order to study and evaluate the large number of concepts that have already been considered. This work can therefore help him to 
make a technological choice during the development process by using one of them, a combination of them or a different solution still unexplored, depending on the performance criteria hierarchy that concerns the surgical application of the device.

\section{Acknowledgements}

The authors would like to thank A. Riwan and M. Boukallel from CEA LIST, M.D.N. Bonnet, cardiovascular and cardiothoracic surgeon, for the numerous discussions around the mini-invasive surgery problematic, and B. Solano, from CEA LIST, who informed us about some newly available commercial products.

\section{References}

[1] J. Abadie, N. Chaillet and C. Lexcellent, Modeling of a new SMA micro-actuator for active endoscopy applications, Mechatronics 19(4) (2009), 437-442.

[2] Y. Bailly and Y. Amirat, Modeling and control of a hybrid continuum active catheter for aortic aneurysm treatment, Proceedings of the 2005 IEEE International Conference on Robotics and Automation, Barcelona, 2005.

[3] G. Bourbon, P. Minotti, P. Langlet, T. Masuzawa and H. Fujita, Three dimensional active microcatheter combining shape memory alloy actuators and direct-drive tubular electrostatic micromotors, Proceedings of the 4th Conference, Micromachined Devices and Components, Santa Clara, 1998.

[4] P. Breedveld, J.S. Scheltes, E.M. Blom and J.E.I. Verheij, A New, Easily miniaturized steerable endoscope, IEEE Engineering in Medicine and Biology Magazine 24(6) (2005), 40-47.

[5] J.K. Chang, S. Chung, Y. Lee, J. Park, S.-K. Lee, S.S. Yang, S.Y. Moon, J. Tschepe, Y. Chee and D.C. Han, Development of endovascular microtools, Journal of Micromechanics and Microengineering 12 (2002), 824-831.

[6] T. Chiba, K. Naganuma, R. Adachi and T. Utsui, Bendable portion of endoscope. US Patent Application Publication 5860914, 1996.

[7] M.B. Cohn, L.S. Crawford, J.M. Wendlandt and S.S. Shankar, Surgical applications of milli-robots, Journal of Robotics Systems 2(6) (1995), 401-416.

[8] T.G. Cooper, D.T. Wallace, S. Chang, S.C. Anderson, D. Williams and S. Manzo, Surgical tool having positively positionable tendon-actuated multi-disk wrist joint. Intuitive Surgical Inc, US Patent Application Publication 6817974B2, 2004.

[9] L.A. Couvillon, Robotic endoscope. SciMed Life Systems, Inc, US Patent Application Publication 6835173, 2004

[10] D.J. Danitz and A. Gold, Articulating mechanism for remote manipulation of a surgical or diagnostic tool. Novare Surgical Systems, Inc, US Patent Application Publication 7090637B2, 2006.

[11] P. Dario, C. Pagetti, N. Troisfontaine, E. Papa, T. Ciuccu, M.C. Carrozza and M. Marcacci, A miniature steerable end-effector for application in an integrated system for computer-assisted arthroscopy, Proceedings of the 1997 IEEE International Conference on Robotics and Automation, Albuquerque, 1997.

[12] T. Dohi, N. Hata, H. Yamashita, A. Iimura and T. Nakazawa, Bending action member. Multi-Slider Linkage Mechanism, Actuator and Manipulator, THK CO. LTD., US Patent Application Publication 0219581A1, 2007.

[13] D.S. Finley and N.T. Nguyen, Surgical robotics, Current Surgery 62(2) (2005), 262-272.

[14] D. Grant and V. Hayward, Design of high strain shape memory alloy actuator, Proceedings of the 1995 IEEE International Conference on Robotics and Automation, Aichi, 1995.

[15] S. Guo, T. Nakamura and T. Fukuda, Micro active guide wire catheter using ICPF actuator, Proceedings of the 4th International Workshop on Advanced Motion Control, Mie, 1996.

[16] Y. Haga, Y. Muyari, T. Mineta, T. Matsunaga, H. Akahori and M. Esashi, Small diameter hydraulic active bending catheter using laser processed super elastic alloy and silicone rubber tube, Proceedings of the 3rd Annual International IEEE EMBS Special Topic Conference on Microtechnologies in Medicine and Biology, Oahu, 2005.

[17] Y. Haga, Y. Tanahashi and M. Esashi, Small diameter active catheter using shape memory alloy, Proceedings of the IEEE 11th Annual Workshop on MEMS, Heidelberg, 1998.

[18] K. Harada, Z. Bo, S. Enosawa, T. Chiba and M.G. Fujie, Bending laser manipulator for intrauterine surgery and viscoelastic model of fetal rat tissue, IEEE International Conference on Robotics \& Automation, 2007.

[19] Hansen Medical-Artisan-Brochure-EU.pdf. [Internet]. 2010, Hansen Medical, [cited 2010]. Available from: http://www. hansenmedical.com/files/Artisan-Brochure-EU.pdf.

[20] D.E. Hegeman, D.J. Danitz, C.D. Hinman and L.J. Alvord, Tool with articulation lock. Novare Surgical Systems Inc, US Patent Application Publication 0255421A1, 2008.

[21] K. Ikuta, T. Hasegawa and S. Daifu, Hyper Redundant Miniature "Hyper Finger" for Remote Minimally Invasive Surgery in Deep Area, Proceedings of the 2003 IEEE International Conference on Robotics and Automation, Taipei, 2003.

[22] K. Ikuta, H. Ichikawa, K. Suzuki and T. Yamamoto, Safety active catheter with multi-segments driven by innovative hydro-pressure micro actuators, 16th Annual International Conference on MEMS, Kyoto, 2003.

[23] K. Ikuta and M. Nokata, Two-lead-wire drive for multi-micro actuators, Proceedings of the 1999 IEEE International Conference on Robotics \& Automation, Detroit, 1999.

[24] K. Ikuta, M. Nokata and S. Aritomi, Biomedical micro robots driven by miniature cybernetics actuator, Proceedings of the IEEE MEMS 1994 Workshop, Oiso, 1994.

[25] K. Ikuta, M. Tsukamoto and S. Hirose, Shape memory alloy servo actuator system with electric resistance feedback and application for active endoscope, Proceedings of the 1988 IEEE International Conference on Robotics and Automation, Philadelphia, 1988.

[26] K. Ikuta, K. Yamamoto and K. Sasaki, Development of remote microsurgery robot and new surgical procedure for deep and narrow space, Proceedings of the 2003 IEEE International Conference on Robotics \& Automation, Taipei, 2003.

[27] B. Kim, Y. Jeong, H.Y. Lim, J.O. Park, A. Menciassi and P. Dario, Functional colonoscope robot system, Proceedings of the 2003 IEEE International Conference on Robotics and Automation, Taipei, 2003 
[28] Laparoscopic Hand Instruments, Covidien Autosuture. [cited 2010]. Available from: http://www.autosuture.com/ autosuture/pageBuilder.aspx?topicID $=41313 \&$ breadcrumbs $=0: 63659$

[29] W. Lee 2006. Surgical Instrument. US Patent Application Publication, 0206101A1, 2010.

[30] A.J. Madhani, Design of teleoperated surgical instruments for minimally invasive surgery, [PhD thesis], Massachusetts Institute of Technology, Cambridge, 1998.

[31] A.J. Madhani and J.K. Salisbury, Wrist mechanism for surgical instrument for performing minimally invasive surgery with enhanced dexterity and sensitivity, Intuitive Surgical Inc, US Patent Application Publication, 5797900, 1998.

[32] M. Menon, A. Tewari, J.O. Peabody, A. Shrivastava, S. Kaul, A. Bhandari and A.K. Hemal, Vattikuti institute prostatectomy, a technique of robotic radical prostatectomy for management of localized carcinoma of the prostate: experience of over 1100 cases, Urol Clin N Am 31(4) (2004), 701-717.

[33] J.P. Merlet, Optimal design for the micro parallel robot MIPS, Proceedings of the 2002 IEEE International Conference on Robotics and Automation, Washington, DC, 2002.

[34] Olympus - CF-2T160I/L [Internet]. 2010. Olympus Medical Systems Europa GmbH. [cited 2010]. Available from: http://www.olympus-europa.com/endoscopy/427_CF2T160I_L.htm.

[35] Olympus - GIF-Q180 [Internet]. 2010. Olympus Medical Systems Europa GmbH. [cited 2010]. Available from: http://www.olympus-europa.com/endoscopy/427_GIFQ180.htm.

[36] T. Ota, A. Degani, D. Schwartzman, B. Zubiate, J. McGarvey, H. Choset and M.A. Zenati, 30th International Conference of the IEEE EMBS, 2008.

[37] T. Ota, A. Degani, Zubiate, A. Wolf, H. Choset, D. Schwartzman and M.A. Zenati, Epicardial atrial ablation using a novel articulated robotic medical probe via percutaneous subxiphoid approach, innovations: Technology \& Techniques in Cardiothoracic \& Vascular Surgery 1(6) (2006), 335340.

[38] K.T. Park and M. Esashi, A multi-link active catheter with polyimide-based integrated CMOS interface circuits, Journal of Microelectromechanical Systems 8(4) (1999), 349357.

[39] S. Park, K. Takemura and T. Maeno, Development of multi-DOF ultrasonic actuators for surgical tools, 32nd International Congress and Exposition on Noise Control Engineering, Seogwipo, 2003.

[40] J. Peirs, D. Reynaerts and H. Van Brussel, A miniature manipulator for integration in a self-propelling endoscope, Sensors and Actuators A 85 (2000), 409-417.

[41] J. Peirs, D. Reynaerts, H. Van Brussel, G. De Gersem and H.W. Tang, Design of an advanced tool guiding system for robotic surgery, Proceedings of the 2003 IEEE International Conference on Robotics \& Automation, Taipei, 2003.

[42] L. Podsedkowski, Robin heart 0,1 and 3 - mechanical construction development, Bulletin of the Polish Academy of Sciences 53(1) (2005), 79-85.

[43] C. Reboulet, Articulation device with parallel structure and remote movement-transmission appliances applying it. ONERA, French Patent 2672836, 1992.
[44] D. Reynaerts, J. Peirs and H. Van Brussel, Shape memory micro-actuation for a gastro-intestinal intervention, Sensors and Actuators A 77(2) (1999), 157-166.

[45] A. Ruzzu, K. Bade, J. Fahrenberg and D. Maas, Positioning system for catheter tips based on an active microvalve system, Journal of Micromechanics and Microengineering 8 (1998), 162-164.

[46] D. Sallé, P. Bidaud and G. Morel, Optimal design of high dexterity modular mis instrument for coronary artery bypass grafting, Proceedings for the 2004 IEEE International Conference on Robotics and Automation, New Orleans, 2004.

[47] J. Szewczyk, V. de Sars, P.H. Bidaud and G. Dumont, An active tubular polyarticulated micro-system for flexible endoscope. International workshop on micro robots, Micro Machines and Systems, Moscow, 1999.

[48] U. Seibold, B. Kübler and G. Hirzinger, Prototype of instrument for minimally invasive surgery with 6-axis force sensing capability, Proceedings of the 2005 IEEE International Conference on Robotics and Automation, Barcelona, 2005.

[49] N. Simaan, R. Taylor and P. Flint, A dexterous system for laryngeal surgery, multi-backbone bending snake-like slaves for teleoperated dexterous surgical manipulation, Proceedings of the 2004 IEEE International Conference on Robotics and Automation, New Orleans, 2004.

[50] K. Suzumori, S. Iikura and H. Tanaka, Flexible microactuator for miniature robots, an investigation of micro structures, sensors, actuators, machines and robots, Proceedings IEEE MEMS 1991, Nara, 1991

[51] H. Takizawa, H. Tosaka, R. Ohta, S. Kaneko and Y. Ueda, Development of a microfine active bending catheter equipped with MIF tactile sensors, Proceedings of the IEEE International Conference on Micro Electro Mechanical Systems, Orlando, 1999

[52] G. Thomann, M. Betemps and T. Redarce, The Development of a bendable colonoscope tip, Proceedings of the 2003 IEEE International Conference on Robotics and Automation, Taipei, 2003

[53] M. Thompson, D. Dickerson, B.S. Bowman, C.F. Kelly, W.W. Malecki, D. Francischelli, M. Stewart, T. Daigle, D. Gubbin, D. Kim, et al., Device and system for surgical dissection and or guidance of other medical devices into body, Medtronic Inc, World International Property Organisation, 089676A1, 2007.

[54] F. Van Meer, A. Giraud, D. Esteve and X. Dollat, A disposable plastic compact wrist for minimally invasive surgical tools, IEEE/RSJ International Conference on Intelligent Robots and Systems, 2005.

[55] D.T. Wallace, S.C. Anderson and S. Manzo, Platform link wrist mechanism. Ituitive Surgical Inc, US Patent Application Publication, 7066926, 2006.

[56] B.D. Weitzner, G.S. Rogers, A. Solbjor, D. Meglan, R. Ailinger, D.L. Brock, W. Lee and D. Driscoll, Robotic medical instrument system. EndoVia Medical Inc, US Patent Application Publication, 0176751A1, 2004.

[57] B.D. Weitzner, S. Rogers and A. Solbjor, Coaxial catheter system. Hansen Medical Inc, US Patent Application Publication 0119824A1, 2008

[58] K. Xu and N. Simaan, Actuation compensation for flexible surgical snake-like robots with redundant remote actuation, Proceedings of the 2006 IEEE International Conference on Robotics and Automation, Orlando, 2006. 
[59] H. Yamashita, A. Limura, E. Aoki, T. Suzuki, T. Nakazawa, E. Kobayashi, M. Hashizume, I. Sakuma and T. Dohi, Development of endoscopic forceps manipulator using multi-slider linkage mechanism, Proceedings of the 1st Asian Symposium on Computer Aided Surgery - Robotic and Image Guided Surgery, Ibaraki, 2005

[60] H. Yamashita, K. Matsumiya, K. Masamune, H. Liao, T. Chiba and T. Dohi, Two-DOFs bending forceps manipulator of 3.5-mm diameter for intrauterine foetus surgery: Feasibility evaluation, International Journal of Computer Assisted Radiology and Surgery 1 (2006), 218-220.

[61] B. Zubiate, A. Degani and H. Choset, Steerable multi linked device having multiple working ports. US Patent Application Publication 0039690A1, 2008. 

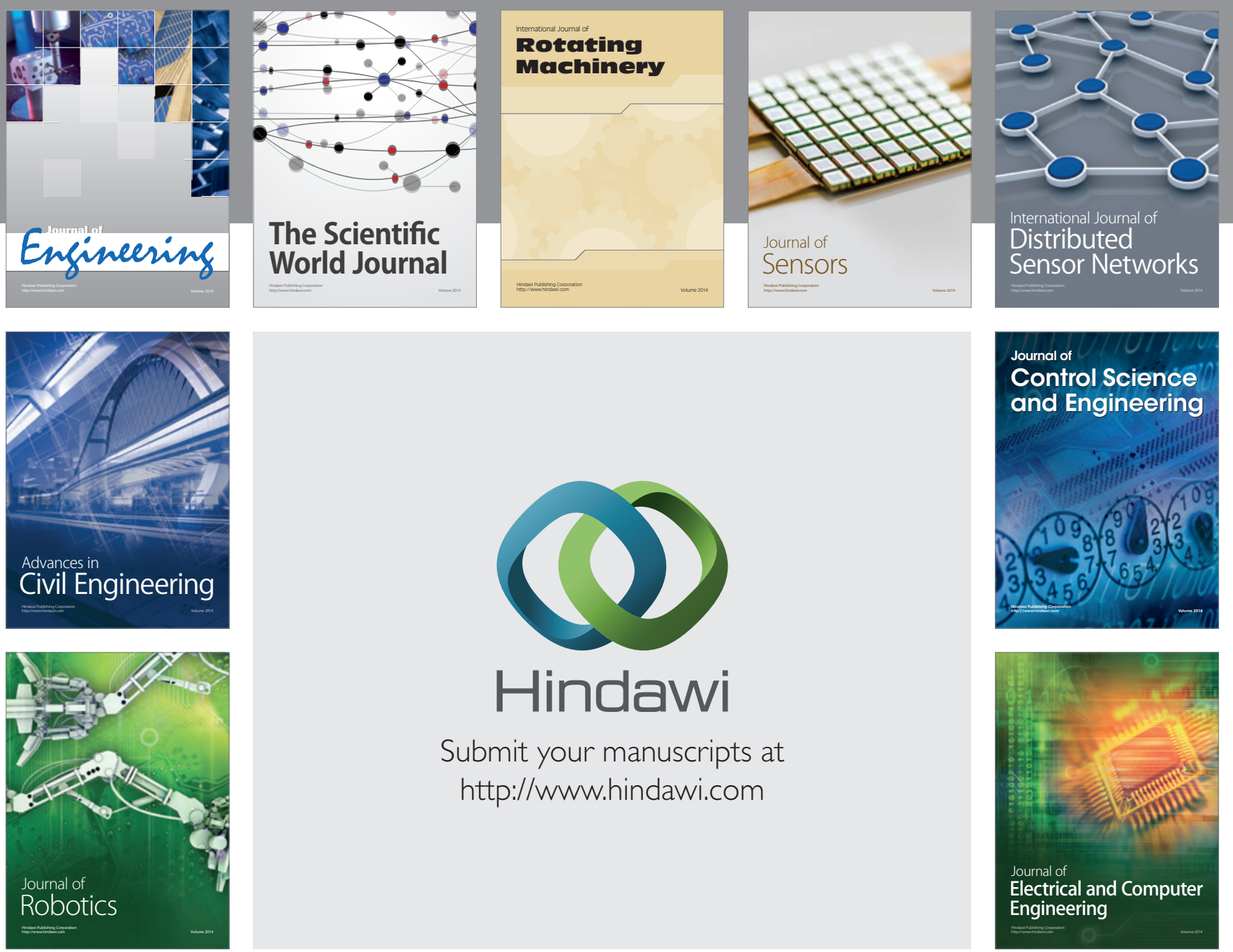

Submit your manuscripts at

http://www.hindawi.com
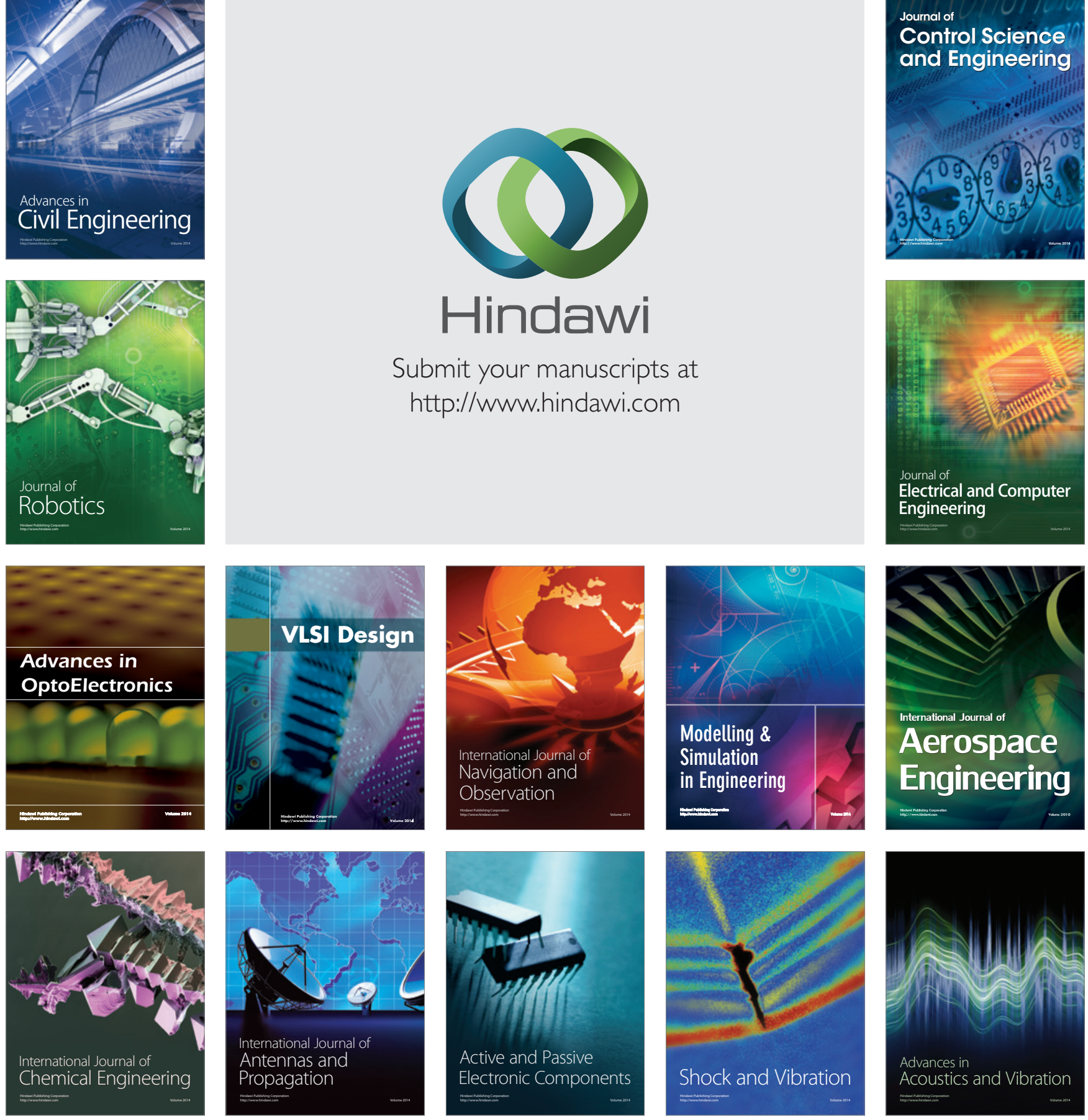